\title{
The multiplier effect in two-sided markets with bilateral investments
}

by Deniz Dizdar, Benny Moldovanu and Nora Szech

No. 109 | NOVEMBER 2017

\section{WORKING PAPER SERIES IN ECONOMICS}

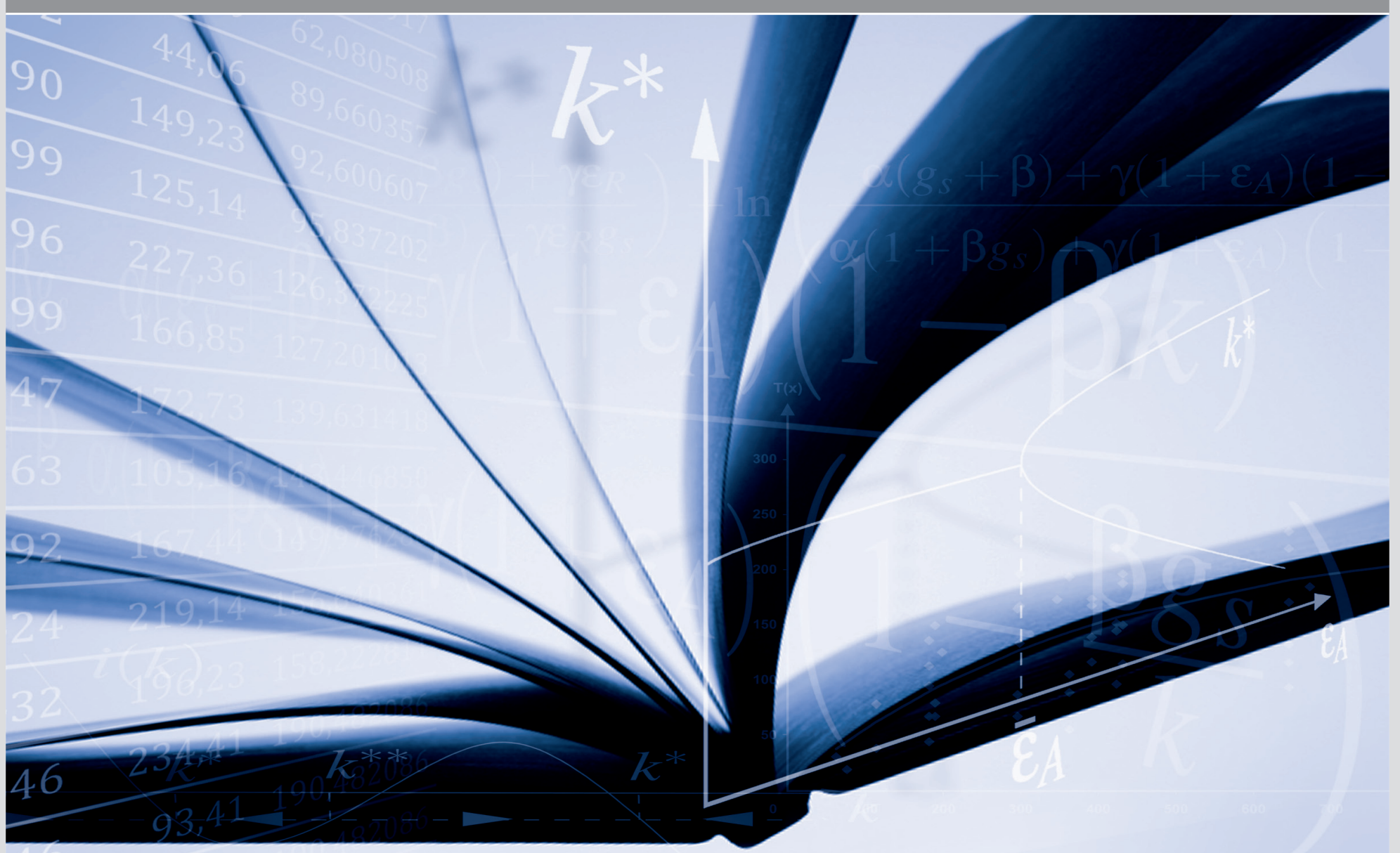




\section{Impressum}

Karlsruher Institut für Technologie (KIT)

Fakultät für Wirtschaftswissenschaften

Institut für Volkswirtschaftslehre (ECON)

Kaiserstraße 12

76131 Karlsruhe

KIT - Die Forschungsuniversität in der Helmholtz-Gemeinschaft

Working Paper Series in Economics

No. 109, November 2017

ISSN 2190-9806

econpapers.wiwi.kit.edu 


\title{
The Multiplier Effect in Two-Sided Markets with Bilateral Investments
}

\author{
Deniz Dizdar, Benny Moldovanu and Nora Szech*
}

November 10, 2017

\begin{abstract}
Agents in a finite two-sided market make costly investments and are then matched assortatively based on these investments. Besides signaling complementary types, investments also generate benefits for partners. We shed light on quantitative properties of the equilibrium investment behavior. The bilateral external benefits induce an investment multiplier effect. This multiplier effect depends in a complex way on agents' uncertainty about their rank within their own market side and on their uncertainty about the types and investments of potential partners. We study how the multiplier effect depends on market size and how it interacts with other important factors such as the costs of investment and the signaling incentives induced by competition for more desirable partners. We use our results to characterize equilibrium utilities in large markets. For small markets, our results lead to bounds on the hold-up problem.
\end{abstract}

JEL Classification: C78, D44, D82

Keywords: matching, signaling, investment, multiplier effect

*Dizdar: Department of Economics, University of Montréal, deniz.dizdar@umontreal.ca. Moldovanu: Department of Economics, University of Bonn, mold@uni-bonn.de. Szech: Department of Economics and Management, Karlsruhe Institute of Technology, nora.szech@kit.edu. Dizdar gratefully acknowledges financial support from the FRQSC. 


\section{Introduction}

In various contexts, individuals, firms or institutions compete for partners in matching markets, and must undertake costly investments prior to matching. These investments render them more attractive to the other side, thus increasing the chance of getting a desirable match. For example, individuals invest resources in education and training with the aim of getting an attractive job, and employers improve their infrastructure in order to attract better qualified and more productive job candidates. The two-sided competition in education markets and in the marriage market, as well as the competition for new business partners, are other examples of this phenomenon.

This paper studies quantitative properties of such bilateral equilibrium investment behavior. We consider two-sided matching contests with incomplete information about complementary productive types, and with arbitrary numbers of participants. In our model, each agent's utility depends on his/her partner's pre-match investment and viceversa, and these bilateral external benefits induce an investment multiplier effect.

The multiplier effect depends in a complex way on agents' uncertainty about their rank in their own population and about the types and investments of potential partners. Nevertheless, we can show that some of its key features are independent of the distributions from which agents' types are drawn. Specifically, the correspondence between investment functions on the two market sides is largely independent of the respective type distributions.

We also quantify how the strength of the multiplier effect depends on market size. Intuitively, in a larger market where agents face less uncertainty about their competitor's types competition intensifies, accompanied by an increased feedback effect of investments. Finally, we analyze the interaction of the multiplier effect with other important factors of the environment such as the costs and benefits of investment, and the signaling incentives induced by the competition for more productive partners.

Two different functions of pre-match investments have been emphasized in the literature. First, in situations in which agents care about underlying, hidden characteristics of their potential partners, privately informed parties may use investments as signals (Spence 1973). Signaling can induce more efficient matchings, but this benefit is partially offset - and may even be outweighed - by the signaling costs (Hoppe, Moldovanu and Sela 2009). On the other hand, in many contexts, agents also derive value from their partner's prior investment, so that investments play a direct productive role (e.g., Peters and Siow 2002; Bhaskar and Hopkins 2016). Most pre-match investments observed in reality have both a signaling and a productive function (Hopkins 2012).

Combining the signaling model of Hoppe, Moldovanu and Sela (2009, henceforth 
HMS) and the investment model of Peters (2007), we study a two-sided matching contest with an NTU (nontransferable utility) matching market and the following main features.

1. There is a finite number of agents on either side of the market, called men and women, respectively. Men and women are characterized by privately known, linearly ordered types that are complementary in the production of output. Consequently, the matching that maximizes aggregate output is positively assortative in types. Signaling can be sustained, as in HMS, without assuming heterogeneity in investment costs. ${ }^{1}$ Types are drawn i.i.d. from two commonly known distributions. We study equilibria in which all agents on the same side of the market use the same, strictly increasing investment strategy so that positively assortative matching based on observable investments implies positively assortative matching of types.

2. Unlike in the HMS model, however, investments are not completely wasteful signals in the present model: they generate benefits for partners that are increasing in the level of investment. As in the complete information setting of Peters (2007), we assume that these benefits enter agents' utilities additively.

For the case in which the external benefits to partners are linear functions of investments, we derive a sharp condition for the existence of a side-symmetric, strictly separating equilibrium, and we find the unique equilibrium in closed form. Existence hinges on the strength of the multiplier effect which is determined by the size of the market in conjunction with the parameters specifying the marginal benefits/costs of investment: since higher investments on one side of the market induce higher investment on the other side and vice-versa, the reinforcing process need not necessarily converge to equilibrium. ${ }^{2}$ For most cases of practical interest, existence is not an issue, but the increases in investment needed to signal small quality differences may still be very large even in markets of moderate size. A related phenomenon currently seems to arise in the case of US colleges. The New York Times speaks of a 'paradox' which in our model, however, occurs in equilibrium: "Typically, fierce market competition leads to lower prices, but among elite schools, the opposite occurs, paradoxically. They often find that raising prices enables them to offer greater benefits to the most coveted potential students. (It also allows them to take part in the amenities race: nicer dorms, better food,

\footnotetext{
${ }^{1}$ The type-independent marginal cost of investment is normalized to 1 .

${ }^{2}$ This is similar to the occurrence of "ruinous" gift exchanges, as documented in the anthropological literature following Boas (1897) and Mauss (1935): "A gives 10 blankets to B; after an interval of time $\mathrm{B}$ gives 20 blankets to A... and so it goes on with the number of blankets being given increasing at a geometric rate" (see Gregory, 1980).
} 
a climbing wall: things that are regarded as essential to attracting those coveted students.)"3 Investments into students' amenities (and students' fees) thus steeply increase across competitors in order to signal (probably much smaller) differences in quality.

We identify the quantity determining the strength of the multiplier effect (in combination with the parameters describing the marginal benefits of investment) as the spectral radius of a matrix whose components are differences among weighted moments of adjacent order statistics from a uniform distribution. Building on the characterization for the case of linear external benefits, we then derive further quantitative results about equilibrium utilities and efficiency properties of investments in the more general model with concave external benefits, distinguishing three cases that correspond to qualitatively different productive roles of investment.

For environments in which investments are "truly" productive in the sense that all Pareto efficient and individually rational investments for a given pair of agents are strictly positive (i.e., exceed the privately optimal investments), we establish lower bounds on equilibrium investments that depend only on the numbers of men and women and on the benefits/costs of investment. These bounds provide novel, quantitative insights into how competition, and in particular how the multiplier effect due to external benefits, alleviate the hold-up problem in small markets with productive investments.

For matching contests in which investments are partially wasteful, we identify the exact asymptotic behavior of equilibrium utilities as the numbers of men and women go to infinity. In those cases, equilibrium utilities converge to those in the unique equilibrium of a continuum model, for which the return to any possible investment is certain. This shows in particular that, even though investments are only partially wasteful, the entire difference between aggregate match surplus and aggregate information rents gets dissipated through competition.

If the marginal benefit from investment is constant and equal to the marginal cost (called below the transferable utility or $T U$ investment case), the continuum model does not even admit a side-symmetric strictly separating equilibrium: the intense competition together with the certainty of returns drive investments to infinity. However, such an equilibrium exists in any finite market with the same characteristics! Our main result for this case demonstrates that in large, balanced markets, the difference between aggregate match surplus and aggregate information rents is always shared fifty-fifty between men and women, irrespectively of other economic aspects such as the shares governing the division of physical surplus in each matched pair and the distributions of types.

\footnotetext{
${ }^{3}$ Davidson, Adam (2015): Is College Tuition Really Too High? In: New York Times, 08 Sept 2015. https://www.nytimes.com/2015/09/13/magazine/is-college-tuition-too-high.html
} 


\section{Related Literature}

Considering one side of the market only, our agents are in a contest situation (see e.g. the survey of Konrad 2007): they compete by means of sunk investments for heterogeneous "prizes," which correspond to matches with the various potential partners. Recognizing this analogy, a sizeable literature has studied pre-match investment problems as matching contests, where agents on both sides of a two-sided market make observable investments and are then matched positive assortatively on the basis of these investments: the highest ranked worker matches with the highest ranked firm, the secondhighest ranked worker matches with the second-highest ranked firm, and so on. In these papers, positively assortative matching based on investments is typically assumed, but it also corresponds to the stable outcome of a frictionless matching market (postinvestment) with nontransferable utility. This is the case for the complete information models of Peters and Siow (2002), Peters (2007), and Bhaskar and Hopkins (2016), in which an agent who invests more generates higher benefits for partners, and also (in equilibrium) for the model of HMS, who assume that investments are completely wasteful signals about hidden characteristics.

Whenever investments generate external benefits, two-sided matching contests are much more complex than standard contests with exogenously given prizes because workers' valuations for the different prizes (i.e., jobs) depend then on firms' investments and vice versa (Peters 2007). This interdependence renders the analysis of noncooperative equilibrium behavior in markets with a finite number of participants (at least two agents on each side of the market) challenging. ${ }^{4}$ With a few important exceptions (Peters 2007, 2011; Bhaskar and Hopkins 2016; Cole, Mailath and Postlewaite 2001b; Felli and Roberts 2016), the literature on pre-match investment problems has circumvented this difficulty by focusing on continuum models in which agents behave competitively (e.g., Cole, Mailath and Postlewaite 2001a; Peters and Siow 2002; Nöldeke and Samuelson 2015; Dizdar 2017).

The work of Peters $(2007,2011)$ demonstrates that non-cooperative equilibrium investments in very large (but finite) two-sided matching contests can be quite different from the investments predicted by a continuum model with competitive agents. More precisely, for models without signaling concerns and with productive investments, Peters shows that equilibrium investments in unbalanced matching contests generally do not converge to competitive (or hedonic) equilibrium investments as the numbers of men and women go to infinity. In particular, agents at the bottom of the distributions

\footnotetext{
${ }^{4}$ Most of the contracting literature has focused on one pair in isolation, e.g. Che and Hausch (1999) who study the hold-up problem in a bilateral contracting situation with cooperative investments that benefit the partner.
} 
generally over-invest. Similar asymptotic results would, a fortiori, apply in our model for unbalanced matching contests in the case of productive investments (compare section 4.2).

We focus instead on a model in which investments also serve as signals, and on developing results about the multiplier effect due to external benefits. This permits new quantitative insights into equilibrium investments and utilities for matching contests with arbitrary numbers of participants, in particular also in markets of small or moderate size.

Bhaskar and Hopkins (2016) study a model with an NTU matching market and noisy investments, building on the tournament model of Lazear and Rosen (1981) rather than on the literature on all-pay contests. Moreover, they assume complete information and that agents on either side of the market are homogenous before they invest. While their main focus is on the analysis of a continuum model, ${ }^{5}$ they also show (under certain conditions) that the corresponding, unique equilibrium is the limit of the non-cooperative equilibria for a finite model. ${ }^{6}$

Olszewski and Siegel (2016) characterize asymptotic bidding behavior in one-sided all-pay contests with many agents and many prizes. Their results allow for complete or incomplete information and for ex-ante asymmetric agents, but because the prize structure is given exogenously these findings cannot be applied to characterize equilibrium behavior in environments with bilateral investments and external benefits. Moreover, their results only hold for very large contests where particular approximation techniques can be applied.

\section{Outline}

The paper is organized as follows. In Section 2, we introduce the model and define various pieces of notation. Section 3 presents the basic equilibrium characterization, the main results about the investment multiplier effect and the closed form solution for the case of linear external benefits. Section 4 contains the remaining results for the cases of partially wasteful, TU or productive investments. All proofs are in the Appendix.

\footnotetext{
${ }^{5}$ In particular, they prove the existence of a unique equilibrium and show that agents over-invest unless the two sides of the market are completely symmetric.

${ }^{6}$ Cole, Mailath and Postlewaite (2001b) and Felli and Roberts (2016) are less directly related to the present study because they analyze models with a TU matching market (i.e., the division of joint surplus is fully flexible) and complete information. Cole, Mailath and Postlewaite (2001b) provide a (non-generic) condition on the ex ante heterogeneity of agents that ensures the existence of a Pareto efficient equilibrium, and they study potential coordination failures due to a form of market incompleteness. Felli and Roberts (2016) characterize the inefficiencies that arise, due to hold-up and coordination problems, when Cole, Mailath and Postlewaite's condition is violated, and when the matching is determined (postinvestment) by a particular bidding game.
} 


\section{Model}

We consider a matching market with $n$ men and $k$ women, where $n \geq k \geq 2$. If $n=k$, we say that the market is balanced. Otherwise, it is unbalanced. Each man is characterized by a privately known type $m \in[\underline{m}, \bar{m}]$, and each woman is characterized by a privately known type $w \in[\underline{w}, \bar{w}]$, where $0 \leq \underline{m}<\bar{m}<\infty$ and $0 \leq \underline{w}<\bar{w}<\infty$. Types are drawn independently from two commonly known, continuous distributions $F$ (for men) and $G$ (for women) with densities $f$ and $g$ that are strictly positive and continuous on $[\underline{m}, \bar{m}]$ and $[\underline{w}, \bar{w}]$, respectively.

All agents simultaneously make costly investments. Men and women are then matched positive assortatively according to their investments: the man with the highest investment is matched to the woman with the highest investment, the man with the second highest investment is matched to the woman with the second highest investment, and so on. Ties are broken randomly.

The net utility of a man with type $m$ and investment $\beta_{M} \in \mathbb{R}_{+}$who is matched to a woman with type $w$ and investment $\beta_{W} \in \mathbb{R}_{+}$is

$$
\gamma_{M} m w+\delta_{M}\left(\beta_{W}\right)-\beta_{M}
$$

and the net utility of the woman in this match is

$$
\gamma_{W} m w+\delta_{W}\left(\beta_{M}\right)-\beta_{W}
$$

Here, $\gamma_{M}>0$ and $\gamma_{W}=1-\gamma_{M}>0$ are constants, and $\delta_{M}: \mathbb{R}_{+} \rightarrow \mathbb{R}_{+}$and $\delta_{W}: \mathbb{R}_{+} \rightarrow \mathbb{R}_{+}$ are non-decreasing, concave, twice continuously differentiable and satisfy $\delta_{M}(0)=0$ and $\delta_{W}(0)=0 .^{7}$ The net utility of an unmatched man (woman) with investment $\beta_{M}$ $\left(\beta_{W}\right)$ is given by $-\beta_{M}\left(-\beta_{W}\right)$.

Note that agents' utility functions are strictly supermodular in their own type and their partner's type, so that investments serve as costly signals in an environment satisfying the standard single-crossing property. HMS's model of assortative matching based on completely wasteful signaling corresponds to the case $\delta_{M}=\delta_{W} \equiv 0$. Following the complete information models of Peters (2007) and Bashkar and Hopkins (2016), the external benefits enter agents' utilities additively, and are modeled via the non-decreasing and type-independent functions $\delta_{M}$ and $\delta_{W} \cdot{ }^{8}$

\footnotetext{
${ }^{7}$ Large parts of our basic equilibrium characterization, including the existence result of Theorem 2 (if the limit conditions are replaced by analogous conditions about limit superiors) apply for arbitrary non-decreasing functions $\delta_{M}$ and $\delta_{W}$, but as all our other quantitative results pertain to linear or concave benefits, we focus on this case from the outset.

${ }^{8} \mathrm{We}$ could easily replace $\gamma_{M} m w$ and $\gamma_{W} m w$ by arbitrary smooth and strictly supermodular functions.
} 


\subsection{Notation}

We need several pieces of notation. $\mathbb{R}_{+}\left(\mathbb{R}_{++}\right)$denotes the set of non-negative (strictly positive) real numbers. We represent vectors in a Euclidean space $\mathbb{R}^{l}, l \in \mathbb{N}$, with respect to a fixed orthonormal basis, and we always label coordinates $0, \ldots, l-1$. $I_{l}$ is the identity on $\mathbb{R}^{l}$, and $\cdot$ is the standard inner product: for $u, v \in \mathbb{R}^{l}, u \cdot v=\sum_{i=0}^{l-1} u_{i} v_{i}$. For $u \in \mathbb{R}^{l},\|u\|_{\infty}$ and $\|u\|_{1}$ denote the vector's maximum norm and $l_{1}$-norm, respectively. ${ }^{9}$ $u>0(u \geq 0)$ means that all entries of $u$ are strictly positive (non-negative). For a matrix $A,\|A\|_{\infty}$ denotes the norm $\max _{u \neq 0} \frac{\|A u\|_{\infty}}{\|u\|_{\infty}}$, and $A>0(A \geq 0)$ means that all entries of $A$ are strictly positive (non-negative).

We let

$$
M_{1: n} \leq M_{2: n} \leq \ldots \leq M_{n: n}
$$

and

$$
W_{1: k} \leq W_{2: k} \leq \ldots \leq W_{k: k}
$$

denote the order statistics of men's and women's types and write $F_{i: n}\left(G_{i: k}\right)$ and $f_{i: n}\left(g_{i: k}\right)$ for the c.d.f. and p.d.f. of $M_{i: n}\left(W_{i: k}\right)$. Thus,

$$
F_{i: n}(m)=\sum_{l=i}^{n}\left(\begin{array}{l}
n \\
l
\end{array}\right) F(m)^{l}(1-F(m))^{n-l}
$$

and

$$
f_{i: n}(m)=n\left(\begin{array}{c}
n-1 \\
i-1
\end{array}\right) F(m)^{i-1}(1-F(m))^{n-i} f(m),
$$

and $G_{i: k}$ and $g_{i: k}$ are given by analogous formulas. For convenience, we also define $M_{0: n} \equiv 0$ and $W_{0: k} \equiv 0$, so that $F_{0: n}=G_{0: k}$ is the c.d.f. of a Dirac measure at 0 .

Next, we define $\mathscr{G}: \mathbb{R} \rightarrow \mathbb{R}^{k}$ as

$$
\mathscr{G}_{j}(w):= \begin{cases}G_{j: k-1}(w) & \text { if } j \in\{1, \ldots, k-1\} \\ 0 & \text { if } j=0 .\end{cases}
$$

For a woman with type $w \in[\underline{w}, \bar{w}], G_{j: k-1}(w)$ is the probability that $j$ or more out of the $k-1$ other women have a type below her own. The entry $\mathscr{G}_{0}(w) \equiv 0$ will be convenient for representing the fact that women do not have to compete for a match with the $k$-th

Such a generalization would require only minor changes to the present analysis. By contrast, weakening additive separability would substantially reduce the analytical tractability of the model.

${ }^{9}$ That is, $\|u\|_{\infty}=\max _{i \in\{0, \ldots, l-1\}}\left|u_{i}\right|$ and $\|u\|_{1}=\sum_{i=0}^{l-1}\left|u_{i}\right|$. 
highest type of man. Similarly, we define $\mathscr{F}: \mathbb{R} \rightarrow \mathbb{R}^{n}$ as

$$
\mathscr{F}_{j}(m):= \begin{cases}F_{j: n-1}(m) & \text { if } j \in\{1, \ldots, n-1\} \\ 0 & \text { if } j=0 .\end{cases}
$$

For any function $h: \mathbb{R} \rightarrow \mathbb{R}$, we define $\Delta_{M}^{h} \in \mathbb{R}^{n}$ as the vector with entries

$$
\Delta_{M, i}^{h}=E\left[h\left(M_{i+1: n}\right)-h\left(M_{i: n}\right)\right], i \in\{0, \ldots, n-1\} .
$$

Similarly, $\Delta_{W}^{h} \in \mathbb{R}^{k}$ is the vector with entries

$$
\Delta_{W, i}^{h}=E\left[h\left(W_{i+1: k}\right)-h\left(W_{i: k}\right)\right], i \in\{0, \ldots, k-1\} .{ }^{10}
$$

\section{Equilibrium Characterization}

In this section, we provide the basic equilibrium characterization, the main quantitative results about the investment multiplier effect, and the closed-form solution for the case of linear benefits. We focus on side-symmetric, strictly separating Bayes-Nash equilibria, i.e., equilibria where all men use the same, strictly increasing strategy and all women use the same, strictly increasing strategy. ${ }^{11}$ Any such equilibrium implements the surplus-maximizing, positive assortative matching of types. We denote equilibrium strategies by $b_{M}:[\underline{m}, \bar{m}] \rightarrow \mathbb{R}_{+}$(for men) and $b_{W}:[\underline{w}, \bar{w}] \rightarrow \mathbb{R}_{+}$(for women).

Suppose that all women use the same, strictly increasing strategy $b_{W}$. Then, the investment game among men is equivalent to an all-pay auction with incomplete information, $n$ ex-ante symmetric bidders and $k$ heterogeneous prizes.

If $n=k$, every man is guaranteed to get at least a match with the worst-ranked partner. For a man with type $m$, the expected utility from this match (the " $k$-th prize") is

$$
\gamma_{M} m E\left[W_{1: k}\right]+E\left[\delta_{M}\left(b_{W}\left(W_{1: k}\right)\right)\right]
$$

The matches with the better-ranked partners then correspond to the $k-1$ prizes that men actually compete for. In particular, for a man with type $m$ the increase in expected utility associated with getting a match with the partner of type $W_{i+1: k}$ rather than with

\footnotetext{
${ }^{10}$ We suppress the dependence on $n$ and $k$ in the notation for various quantities, such as $\Delta_{M}^{h}, \Delta_{W}^{h}$. We will add superscripts in Section 4.1, where we study sequences of matching contests of different sizes.

${ }^{11}$ If $n>k$, strict supermodularity and the fact that men want to avoid staying unmatched ensures that all side-symmetric equilibria are strictly separating (given that positive assortative matching is assumed as part of the game). If $n=k$, there is also a side-symmetric equilibrium in which nobody invests.
} 
$W_{i: k}, i \in\{1, \ldots, k-1\}$, is

$$
\gamma_{M} m E\left[W_{i+1: k}-W_{i: k}\right]+E\left[\delta_{M}\left(b_{W}\left(W_{i+1: k}\right)\right)-\delta_{M}\left(b_{W}\left(W_{i: k}\right)\right)\right],
$$

which is strictly increasing in $m$ (strict single crossing). Note how the prizes here are endogenous and depend on the strategy employed by women. This is the defining characteristic of two-sided contests with investments that generate external benefits.

If $n>k$, men also have to compete for the " $k$-th prize". In either case, existing results for all-pay auctions with incomplete information and with ex-ante symmetric bidders imply that the contest among men has a unique symmetric equilibrium. Moreover, the strictly increasing, differentiable equilibrium strategy can be derived by the standard first-order approach. ${ }^{12}$ An analogous argument applies for women, who are guaranteed to get at least a match with the $k$-th ranked man, $M_{n-k+1: n}$.

Thus, a side-symmetric, strictly separating equilibrium of the matching contest corresponds to a pair of functions: $b_{M}$ is the symmetric equilibrium strategy of the all-pay auction among men for which the prizes are determined by the order statistics induced by $G$ and by the women's strategy $b_{W}$, and $b_{W}$ is the symmetric equilibrium strategy of the all-pay auction among women for which the prizes are induced by the order statistics of $F$ and by the men's strategy $b_{M}$. In equilibrium, the types are matched positive assortatively: for $i \in\{1, \ldots, k\}$, the man with type $M_{n-k+i: n}$ is matched to the woman with type $W_{i: k}$.

A man with type $\underline{m}$ gets the worst possible match with probability 1 in equilibrium, ${ }^{13}$ which implies $b_{M}(\underline{m})=0 .{ }^{14}$ Similarly, $b_{W}(\underline{w})=0 .{ }^{15}$ For a man with type $m>\underline{m}$ who assumes that all other agents use strictly increasing, differentiable strategies $b_{M}$ and $b_{W}$, the problem of maximizing his expected utility is to choose an $s \in[\underline{m}, \bar{m}]$ that maximizes

$$
\begin{aligned}
& \sum_{j=0}^{k-1} \mathscr{F}_{n-k+j}(s) E\left[\gamma_{M} m\left(W_{j+1: k}-W_{j: k}\right)+\delta_{M}\left(b_{W}\left(W_{j+1: k}\right)\right)-\delta_{M}\left(b_{W}\left(W_{j: k}\right)\right)\right]-b_{M}(s) \\
& =\hat{\mathscr{F}}(s) \cdot\left(\gamma_{M} m \Delta_{W}^{I_{1}}+\Delta_{W}^{\delta_{M} \circ b_{W}}\right)-b_{M}(s)
\end{aligned}
$$

where we have used the following notation.

Definition 1. For $u \in \mathbb{R}^{n}$, let $\hat{u} \in \mathbb{R}^{k}$ denote the vector with entries $\hat{u}_{i}=u_{n-k+i}$, $i \in$ $\{0, \ldots, k-1\}$.

\footnotetext{
${ }^{12}$ Sufficient conditions are known to be satisfied.

${ }^{13}$ If $n=k$, he is matched to the woman with type $W_{1: k}$. If $n>k$, he stays unmatched.

${ }^{14}$ If $b_{M}(\underline{m})$ were strictly positive, type $\underline{m}$ could decrease his investment without changing his expected match.

${ }^{15}$ If $\underline{w}>0$, we also formally set $b_{W}(0)=0$ (so that $b_{W}\left(W_{0: k}\right)=0$ ).
} 
In equilibrium, the first order condition must be satisfied at $s=m$, i.e.,

$$
b_{M}^{\prime}(m)=\hat{\mathscr{F}}^{\prime}(m) \cdot\left(\gamma_{M} m \Delta_{W}^{I_{1}}+\Delta_{W}^{\delta_{M} \circ b_{W}}\right) .
$$

Integrating the above, we obtain

$$
b_{M}(m)=\gamma_{M} a_{M}(m)+\hat{\mathscr{F}}(m) \cdot \Delta_{W}^{\delta_{M} \circ b_{W}},
$$

where

$$
a_{M}(m)=\left(m \hat{\mathscr{F}}(m)-\int_{\underline{m}}^{m} \hat{\mathscr{F}}(s) d s\right) \cdot \Delta_{W}^{I_{1}} \cdot{ }^{16}
$$

An analogous derivation yields

$$
b_{W}(w)=\gamma_{W} a_{W}(w)+\mathscr{G}(w) \cdot \hat{\Delta}_{M}^{\delta_{W} \circ b_{M}}
$$

where

$$
a_{W}(w)=\left(w \mathscr{G}(w)-\int_{\underline{w}}^{w} \mathscr{G}(s) d s\right) \cdot \hat{\Delta}_{M}^{I_{1}} .
$$

In particular, using (4) we have for all $i \in\{0, \ldots, n-1\}$ :

$$
\begin{aligned}
\Delta_{M, i}^{\delta_{W} \circ b_{M}} & =E\left[\delta_{W}\left(\gamma_{M} a_{M}\left(M_{i+1: n}\right)+\hat{\mathscr{F}}\left(M_{i+1: n}\right) \cdot \Delta_{W}^{\delta_{M} \circ b_{W}}\right)\right. \\
& \left.-\delta_{W}\left(\gamma_{M} a_{M}\left(M_{i: n}\right)+\hat{\mathscr{F}}\left(M_{i: n}\right) \cdot \Delta_{W}^{\delta_{M} \circ b_{W}}\right)\right] .
\end{aligned}
$$

Similarly, (5) implies for all $i \in\{0, \ldots, k-1\}$ :

$$
\begin{aligned}
\Delta_{W, i}^{\delta_{M} \circ b_{W}} & =E\left[\delta_{M}\left(\gamma_{W} a_{W}\left(W_{i+1: k}\right)+\mathscr{G}\left(W_{i+1: k}\right) \cdot \hat{\Delta}_{M}^{\delta_{W} \circ b_{M}}\right)\right. \\
& \left.-\delta_{M}\left(\gamma_{W} a_{W}\left(W_{i: k}\right)+\mathscr{G}\left(W_{i: k}\right) \cdot \hat{\Delta}_{M}^{\delta_{W} \circ b_{M}}\right)\right] .
\end{aligned}
$$

Defining $T: \mathbb{R}_{+}^{k} \rightarrow \mathbb{R}_{+}^{n}$ and $S: \mathbb{R}_{+}^{k} \rightarrow \mathbb{R}_{+}^{k}$ via

$$
\begin{aligned}
& T_{i}(y):=E\left[\delta_{W}\left(\gamma_{M} a_{M}\left(M_{i+1: n}\right)+\hat{\mathscr{F}}\left(M_{i+1: n}\right) \cdot y\right)-\delta_{W}\left(\gamma_{M} a_{M}\left(M_{i: n}\right)+\hat{\mathscr{F}}\left(M_{i: n}\right) \cdot y\right)\right], \\
& S_{i}(x):=E\left[\delta_{M}\left(\gamma_{W} a_{W}\left(W_{i+1: k}\right)+\mathscr{G}\left(W_{i+1: k}\right) \cdot x\right)-\delta_{M}\left(\gamma_{W} a_{W}\left(W_{i: k}\right)+\mathscr{G}\left(W_{i: k}\right) \cdot x\right)\right],
\end{aligned}
$$

we obtain the following fixed point characterization of side-symmetric, strictly separating equilibria of the matching contest. ${ }^{17}$

\footnotetext{
${ }^{16}$ Note that $\gamma_{M} a_{M}$ is actually the men's equilibrium strategy in the HMS special case where women's investments create no benefits for men $\left(\delta_{M} \equiv 0\right)$.

${ }^{17} T$ maps $\mathbb{R}_{+}^{k}$ into $\mathbb{R}_{+}^{n}$ because $\delta_{W}, a_{M}$ and all coordinate functions of $\hat{\mathscr{F}}$ are non-decreasing. An analogous observation applies to $S$.
} 
Lemma 1. The mapping $\imath: b_{W} \mapsto \Delta_{W}^{\delta_{M} \circ b_{W}}$ is a bijection between the set of side-symmetric, strictly separating equilibria and the set of fixed points of $S \circ \hat{T} .{ }^{18}$

The logic behind this fixed point argument is as follows. The equilibrium strategies determine the vectors of increments in the matching benefits $\Delta_{W}^{\delta_{M} \circ b_{W}}$ and $\hat{\Delta}_{M}^{\delta_{W} \circ b_{M}}$. Conversely, these increment vectors are sufficient to recover the strategies through (4) and (5). This is due to the fact that the increment vector of each market side determines the "prize structure" of the all-pay auction played by the other market side. In particular, the mappings $S$ and $\hat{T}$ capture how any increment vector for one market side pins down a unique increment vector for the other side. In equilibrium, the pair of male and female increment vectors must be consistent with each other. In other words, $\Delta_{W}^{\delta_{M} \circ b_{W}}$ is a fixed point of $S \circ \hat{T}$ and $\hat{\Delta}_{M}^{\delta_{W} \circ b_{M}}$ is a fixed point of $\hat{T} \circ S$.

We now turn to the observation that will allow quantitative insights into equilibrium investment behavior and interim expected utilities. Men face the kind of uncertainty regarding the types of their competitors (and hence about which of the $k$ heterogeneous prizes they will win with any particular investment) that is standard in auctions or in contests with one-dimensional heterogeneity and ex-ante symmetric agents. Interestingly, key aspects of how the heterogeneity of the prizes affects the investment increments given by

$$
\begin{aligned}
\Delta_{M, i}^{b_{M}} & =E\left[b_{M}\left(M_{i+1: n}\right)-b_{M}\left(M_{i: n}\right)\right] \\
& =\gamma_{M} \Delta_{M, i}^{a_{M}}+\sum_{j=0}^{k-1} E\left[F_{n-k+j: n-1}\left(M_{i+1: n}\right)-F_{n-k+j: n-1}\left(M_{i: n}\right)\right] \Delta_{W, j}^{\delta_{M} \circ b_{W}},
\end{aligned}
$$

turn out to be independent of the distribution $F$ (an analogous observation applies for women, of course).

To see this, note that $F\left(M_{i: n}\right)$ is distributed like the $i$-th order statistic of $n$ independent draws from the uniform distribution $U(0,1)$ (see, for example, Theorem 1.2.5 in Reiss 1989), and that $F_{j: n-1}$ is a polynomial in $F$. In particular, $E\left[F_{j: n-1}\left(M_{i: n}\right)\right]$ does not depend on the distribution $F$ (the term is a weighted sum of moments of the $\mathrm{i}$-th order statistic of $n$ independent draws from $U(0,1))$. This motivates the following definitions.

\section{Definition 2.}

1. Let $\Theta_{n} \in \mathbb{R}^{n \times n}$ be the matrix with entries $\theta_{n, i j}=E\left[F_{j: n-1}\left(M_{i+1: n}\right)-F_{j: n-1}\left(M_{i: n}\right)\right]$ for $i, j \in\{0, \ldots, n-1\}$. Let $\Theta_{n, k} \in \mathbb{R}^{n \times k}$ be the matrix that results from deleting

\footnotetext{
${ }^{18}$ Note that if $b_{W}$ is an equilibrium strategy, the equilibrium strategy for men is uniquely determined by (4).
} 
the first $n-k$ columns of $\Theta_{n}$, and let $\hat{\Theta}_{n, k} \in \mathbb{R}^{k \times k}$ be the matrix that results from deleting the first $n-k$ rows of $\Theta_{n, k} \cdot{ }^{19}$

2. Let $r(n, k)>0$ be the real eigenvalue of the matrix $\Theta_{k} \hat{\Theta}_{n, k}$ satisfying $r(n, k) \geq|\lambda|$ for any other eigenvalue $\lambda$ of $\Theta_{k} \hat{\Theta}_{n, k}$.

The existence of $r(n, k)$ follows because $\Theta_{k} \hat{\Theta}_{n, k}$ is a non-zero and non-negative matrix (see Theorems 8.1.22 and 8.3.1 in Horn and Johnson 2013). The following lemma provides the explicit form of $\Theta_{n}$ and establishes a somewhat surprising fact: $\frac{n+1}{n-1} \Theta_{n}$ is a stochastic matrix.

Lemma 2. i) For all $i \in\{0, \ldots, n-1\}$,

$$
\theta_{n, i j}= \begin{cases}0 & \text { if } j=0 \\
\frac{n-1}{2 n-1} \frac{\left(\begin{array}{c}
n \\
i
\end{array}\right)\left(\begin{array}{c}
n-2 \\
j-1
\end{array}\right)}{\left(\begin{array}{c}
2 n-2 \\
i+j-1
\end{array}\right)} & \text { if } j \in\{1, \ldots, n-1\} .\end{cases}
$$

ii) $\frac{n+1}{n-1} \Theta_{n}$ is a (row-)stochastic matrix.

Armed with Definition 2, we can write (8) in the following compact form:

$$
\Delta_{M}^{b_{M}}=\gamma_{M} \Delta_{M}^{a_{M}}+\Theta_{n, k} \Delta_{W}^{\delta_{M} \circ b_{W}}
$$

Similarly,

$$
\Delta_{W, i}^{b_{W}}=\gamma_{W} \Delta_{W, i}^{a_{W}}+\sum_{j=0}^{k-1} E\left[G_{j: k-1}\left(W_{i+1: k}\right)-G_{j: k-1}\left(W_{i: k}\right)\right] \hat{\Delta}_{M, j}^{\delta_{W} \circ b_{M}}
$$

implies

$$
\Delta_{W}^{b_{W}}=\gamma_{W} \Delta_{W}^{a_{W}}+\Theta_{k} \hat{\Delta}_{M}^{\delta_{W} \circ b_{M}} .
$$

Thus, the matrix $\Theta_{n, k}\left(\Theta_{k}\right)$ captures how the type-independent increments in expected benefits that men (women) obtain from getting partners with higher investments translate into investment increments (Lemma 4 in the Appendix shows that these distributionindependent matrices also occur in the explicit representations of $\Delta_{M}^{a_{M}}$ and $\Delta_{W}^{a_{W}}$, i.e., they are also important for computing bid increments for the standard all pay-auction model with heterogenous prizes, where an agent's valuation is multiplicative in his/her type and the prize he/she gets).

If the benefit functions are linear, i.e., if $\delta_{M}\left(\beta_{W}\right)=d_{M} \beta_{W}$ and $\delta_{W}\left(\beta_{M}\right)=d_{W} \beta_{M}$ for constants $d_{M}, d_{W} \geq 0$, (9) and (10) allow us to precisely characterize the strength

\footnotetext{
${ }^{19}$ That is, the entries of $\hat{\Theta}_{n, k}$ are $\theta_{n,(n-k+i)(n-k+j)}$, for $i, j \in\{0, \ldots, k-1\}$.
} 
of the multiplier effect, to obtain a necessary and sufficient condition for equilibrium existence that depends only on $n, k, d_{M}$ and $d_{W}$, and to derive the unique equilibrium in closed form.

Theorem 1 (Equilibrium characterization for linear benefits). Assume that $\delta_{M}\left(\beta_{W}\right)=$ $d_{M} \beta_{W}$ and $\delta_{W}\left(\beta_{M}\right)=d_{W} \beta_{M}$ for constants $d_{M}, d_{W} \geq 0$. A side-symmetric, strictly separating equilibrium exists if and only if $d_{M} d_{W} r(n, k)<1$. If it exists, the equilibrium is unique. The equilibrium strategies satisfy

$$
\begin{aligned}
& b_{M}(m)=\gamma_{M} a_{M}(m)+d_{M} \hat{\mathscr{F}}(m) \cdot \Delta_{W}^{b_{W}} \\
& b_{W}(w)=\gamma_{W} a_{W}(w)+d_{W} \mathscr{G}(w) \cdot \hat{\Delta}_{M}^{b_{M}}
\end{aligned}
$$

where $\Delta_{W}^{b_{W}}$ and $\hat{\Delta}_{M}^{b_{M}}$ are explicitly given by

$$
\begin{aligned}
\hat{\Delta}_{M}^{b_{M}} & =\left(I_{k}-d_{M} d_{W} \hat{\Theta}_{n, k} \Theta_{k}\right)^{-1}\left(\gamma_{M} \hat{\Delta}_{M}^{a_{M}}+d_{M} \gamma_{W} \hat{\Theta}_{n, k} \Delta_{W}^{a_{W}}\right) \\
\Delta_{W}^{b_{W}} & =\left(I_{k}-d_{M} d_{W} \Theta_{k} \hat{\Theta}_{n, k}\right)^{-1}\left(\gamma_{W} \Delta_{W}^{a_{W}}+d_{W} \gamma_{M} \Theta_{k} \hat{\Delta}_{M}^{a_{M}}\right) .
\end{aligned}
$$

Equilibrium existence for $r(n, k) d_{M} d_{W}<1$ and the closed form follow easily from (9), (10) and from $r(n, k)$ being the spectral radius of $\Theta_{k} \hat{\Theta}_{n, k}$. The condition $r(n, k) d_{M} d_{W}<$ 1 roughly says that the marginal feedback benefit of an additional unit of investment that combines the two marginal external benefits from investment $d_{M}$ and $d_{W}$ and the multiplier $r(n, k)$ - is less than the marginal cost of investment, which is normalized here to unity. The proof that no equilibrium exists when the condition does not hold is based on Farkas' Lemma and uses the left eigenvector associated with the eigenvalue $r(n, k)$.

Remark 1. Lemma 2 implies $r(n, n)=\frac{(n-1)^{2}}{(n+1)^{2}}$, so that we immediately obtain the exact strength of the multiplier effect in all balanced markets. ${ }^{20}$ For $n>k$, we obtain that $r(n, k) \leq \frac{(n-1)(k-1)}{(n+1)(k+1)}$ because $\frac{(n+1)(k+1)}{(n-1)(k-1)} \Theta_{k} \hat{\Theta}_{n, k}$ is sub-stochastic. Moreover, $r(n, k)$ can easily be computed numerically using the explicit form of $\Theta_{n}{ }^{21}$

For the case of general benefit functions, we establish equilibrium existence using Brouwer's Fixed Point Theorem.

Theorem 2. If $\frac{(n-1)(k-1)}{(n+1)(k+1)}\left(\lim _{\beta_{W} \rightarrow \infty} \delta_{M}^{\prime}\left(\beta_{W}\right)\right)\left(\lim _{\beta_{M} \rightarrow \infty} \delta_{W}^{\prime}\left(\beta_{M}\right)\right)<1$, then there exists a side-symmetric, strictly separating equilibrium.

Of course, equilibrium strategies always satisfy (4) and (5), and investment increments always satisfy (9) and (10).

\footnotetext{
${ }^{20}$ Note that for $n=k, \Theta_{k}=\hat{\Theta}_{n, k}=\Theta_{n}$.

${ }^{21}$ For $n>k, \Theta_{k} \hat{\Theta}_{n, k}>0$, so that, by Perron's Theorem, $r(n, k)>|\lambda|$ for all other eigenvalues $\lambda$. This makes the numerical computation of $r(n, k)$ particularly straightforward.
} 


\subsection{An Illustration: the $2 \times 2$ case}

We briefly consider here the balanced case with two agents on each side of the market, and where $\delta_{M}\left(\beta_{W}\right)=d_{M} \beta_{W}$ and $\delta_{W}\left(\beta_{M}\right)=d_{W} \beta_{M}$ for constants $d_{M}, d_{W} \geq 0$. We have

$$
\Theta_{2}=\left(\begin{array}{cc}
0 & \frac{1}{3} \\
0 & \frac{1}{3}
\end{array}\right)
$$

so that $r(2,2)$, the spectral radius of $\Theta_{2}^{2}$, is equal to $\frac{1}{9}$. Thus, a side-symmetric, strictly separating equilibrium exists if and only if $d_{M} d_{W}<9$. Moreover, we obtain that

$$
\left(I_{2}-d_{M} d_{W} \Theta_{2}^{2}\right)^{-1}=\left(\begin{array}{cc}
1 & -\frac{d_{M} d_{W}}{9-d_{M} d_{W}} \\
0 & \frac{9}{9-d_{M} d_{W}}
\end{array}\right) .
$$

The equilibrium strategies satisfy

$$
b_{M}(m)=\gamma_{M} a_{M}(m)+d_{M} \mathscr{F}(m) \cdot \Delta_{W}^{b_{W}}=\gamma_{M} a_{M}(m)+d_{M} F(m) \Delta_{W, 1}^{b_{W}}
$$

and

$$
b_{W}(w)=\gamma_{W} a_{W}(w)+d_{W} \mathscr{G}(w) \cdot \Delta_{M}^{b_{M}}=\gamma_{W} a_{W}(w)+d_{W} G(w) \Delta_{M, 1}^{b_{M}},
$$

where $\Delta_{W}^{b_{W}}$ and $\Delta_{M}^{b_{M}}$ are explicitly given by

$$
\begin{aligned}
& \Delta_{M}^{b_{M}}=\left(\begin{array}{cc}
1 & -\frac{d_{M} d_{W}}{9-d_{M} d_{W}} \\
0 & \frac{9}{9-d_{M} d_{W}}
\end{array}\right)\left(\gamma_{M} \Delta_{M}^{a_{M}}+d_{M} \gamma_{W}\left(\begin{array}{cc}
0 & \frac{1}{3} \\
0 & \frac{1}{3}
\end{array}\right) \Delta_{W}^{a_{W}}\right) \\
& \Delta_{W}^{b_{W}}=\left(\begin{array}{cc}
1 & -\frac{d_{M} d_{W}}{9-d_{M} d_{W}} \\
0 & \frac{9}{9-d_{M} d_{W}}
\end{array}\right)\left(\gamma_{W} \Delta_{W}^{a_{W}}+d_{W} \gamma_{M}\left(\begin{array}{cc}
0 & \frac{1}{3} \\
0 & \frac{1}{3}
\end{array}\right) \Delta_{M}^{a_{M}}\right) .
\end{aligned}
$$

In particular, the relevant, second-row entries (with index 1) are given by

$$
\Delta_{M, 1}^{b_{M}}=\frac{9}{9-d_{M} d_{W}}\left(\gamma_{M} \Delta_{M, 1}^{a_{M}}+d_{M} \gamma_{W} \frac{1}{3} \Delta_{W, 1}^{a_{W}}\right)
$$

and

$$
\Delta_{W, 1}^{b_{W}}=\frac{9}{9-d_{M} d_{W}}\left(\gamma_{W} \Delta_{W, 1}^{a_{W}}+d_{W} \gamma_{M} \frac{1}{3} \Delta_{M, 1}^{a_{M}}\right) .
$$

To explicitly pin down the equilibrium strategies, we thus only need to identify the terms $\Delta_{M, 1}^{a_{M}}$ and $\Delta_{W, 1}^{a_{W}}$. From Lemma 4 in the Appendix we obtain

$$
\Delta_{M, 1}^{a_{M}}=\frac{1}{3} E\left[M_{2: 3}\right] E\left[W_{2: 2}-W_{1: 2}\right] \quad \text { and } \quad \Delta_{W, 1}^{a_{W}}=\frac{1}{3} E\left[W_{2: 3}\right] E\left[M_{2: 2}-M_{1: 2}\right]
$$


For the remainder of this example, we assume that $0<d=d_{M}=d_{W}<1$ and that $F=G$. We show that if $F$ has "increasing virtual values", i.e., $m-\frac{1-F(m)}{f(m)}$ is increasing, and if $\underline{m}=0$, then $E\left[b_{M}\left(M_{1: 2}\right)\right]$ is strictly increasing in $\gamma_{M}$. Since $d E\left[b_{M}\left(M_{1: 2}\right)\right]$ equals the expected utility of a woman with type zero (see Lemma 3 ), this implies, by continuity of the distribution and of the bid functions, that there is a positive measure of (low) women types whose expected utility decreases when the match share $\gamma_{W}=1-\gamma_{M}$ of women increases! As these types get most of their utility from their partner's investments that may be relatively high (rather than from the jointly produced matching output, which is low) an increase in women's share of output leads to a decrease in men's investments, and hence to a decrease in the expected utility of low women types. Here is the argument.

Proof. Note that $E\left[F\left(M_{1: 2}\right)\right]=\theta_{2,01}=\frac{1}{3}$ and that $E\left[a_{M}\left(M_{1: 2}\right)\right]=\frac{1}{3} E\left[M_{1: 3}\right] E\left[W_{2: 2}-\right.$ $W_{1: 2}$ (by Lemma 4 in the Appendix). From this, (13), (14), (15) and the assumptions that $F=G$ and $d=d_{M}=d_{W}$, we immediately obtain

$$
E\left[b_{M}\left(M_{1: 2}\right)\right]=\left(\gamma_{M} E\left[M_{1: 3}\right]+d \frac{3 \gamma_{W}+d \gamma_{M}}{9-d^{2}} E\left[M_{2: 3}\right]\right) \frac{E\left[M_{2: 2}-M_{1: 2}\right]}{3} .
$$

Since we are interested in the dependence on $\gamma_{M}$, we substitute $\gamma_{W}=1-\gamma_{M}$ and write

$$
E\left[b_{M}\left(M_{1: 2}\right)\right]=\left(\frac{3 d}{9-d^{2}} E\left[M_{2: 3}\right]+\gamma_{M}\left(E\left[M_{1: 3}\right]-\frac{d}{3+d} E\left[M_{2: 3}\right]\right)\right) \frac{E\left[M_{2: 2}-M_{1: 2}\right]}{3}
$$

Thus, $E\left[b_{M}\left(M_{1: 2}\right)\right]$ is strictly increasing in $\gamma_{M}$ if and only if $\left(\frac{3}{d}+1\right) E\left[M_{1: 3}\right]>E\left[M_{2: 3}\right]$. Lemma 5 in the Appendix shows that the assumption of increasing virtual valuations implies $4 E\left[M_{1: 3}\right] \geq E\left[M_{2: 3}\right]$. This completes the proof.

\subsection{Equilibrium Utility}

Given the separable form of the utility functions, we can use the standard payoff equivalence result for Bayesian incentive compatible mechanisms to represent interim expected utilities. For a side-symmetric, strictly separating equilibrium, we let $U_{M}(m)$ denote the expected utility for a man with type $m$, and $U_{W}(w)$ denote the expected utility for a woman with type $w$. We write $\Psi(m) \in[0, \bar{w}]$ for type $m$ 's expected type of 
partner and $\Phi(w) \in[\underline{m}, \bar{m}]$ for type $w$ 's expected type of partner. Thus,

$$
\begin{aligned}
& \Psi(m)= \begin{cases}E\left[W_{1: n}\right]+\mathscr{F}(m) \cdot \Delta_{W}^{I_{1}} & \text { if } n=k \\
\hat{\mathscr{F}}(m) \cdot \Delta_{W}^{I_{1}} & \text { if } n>k .\end{cases} \\
& \Phi(w)=E\left[M_{n-k+1: n}\right]+\mathscr{G}(w) \cdot \hat{\Delta}_{M}^{I_{1}} .
\end{aligned}
$$

Lemma 3. Agents' interim expected utilities satisfy:

$$
\begin{aligned}
& U_{M}(m)= \begin{cases}E\left[\delta_{M}\left(b_{W}\left(W_{1: n}\right)\right)\right]+\gamma_{M} \underline{m} E\left[W_{1: n}\right]+\gamma_{M} \int_{\underline{m}}^{m} \Psi(s) d s & \text { if } n=k \\
\gamma_{M} \int_{\underline{m}}^{m} \Psi(s) d s & \text { if } n>k .\end{cases} \\
& U_{W}(w)=E\left[\delta_{W}\left(b_{M}\left(M_{n-k+1: n}\right)\right)\right]+\gamma_{W} \underline{w} E\left[M_{n-k+1: n}\right]+\gamma_{W} \int_{\underline{w}}^{w} \Phi(s) d s .
\end{aligned}
$$

For completeness, an explicit proof of Lemma 3 is included in the Appendix. In the next section, we contrast these interim expected utilities with their counterparts in the model with a continuum of agents.

\section{The Productive Role of Investments}

In this section, we develop further quantitative results about equilibrium investments and expected utilities. We distinguish three cases that correspond to three fundamentally different productive roles of agents' investments.

1. (Partially) wasteful investments: $\delta_{M}^{\prime}(0) \delta_{W}^{\prime}(0)<1$.

2. Monetary transfers under quasi-linear utility (TU): $\delta_{M}\left(\beta_{W}\right)=\beta_{W}$ and $\delta_{W}\left(\beta_{M}\right)=\beta_{M}$.

3. Productive investments: $\delta_{M}^{\prime}(0) \delta_{W}^{\prime}(0)>1$ and $\lim _{\beta_{W} \rightarrow \infty} \delta_{M}^{\prime}\left(\beta_{W}\right) \lim _{\beta_{M} \rightarrow \infty} \delta_{W}^{\prime}\left(\beta_{M}\right)<1$.

By Theorem 2, a side-symmetric, strictly separating equilibrium exists for all three cases.

If $\delta_{M}^{\prime}(0)>0$ and $\delta_{W}^{\prime}(0)>0$ investments generate benefits for partners. Yet, if $\delta_{M}^{\prime}(0) \delta_{W}^{\prime}(0)<1$, positive investments are still partially wasteful. In this case, the only pair of Pareto efficient and individually rational investments for a given pair of agents ${ }^{22}$ is $\left(\beta_{M}, \beta_{W}\right)=(0,0)$, and all $\left(\beta_{M}, \beta_{W}\right) \in R_{++}^{2}$ are inefficient.

In the transferable utility case in which $\delta_{M}\left(\beta_{W}\right)=\beta_{W}$ and $\delta_{W}\left(\beta_{M}\right)=\beta_{M}$, all possible $\left(\beta_{M}, \beta_{W}\right)$ are Pareto efficient for a given pair. By contrast, if $\delta_{M}^{\prime}(0) \delta_{W}^{\prime}(0)>1$,

\footnotetext{
${ }^{22}$ Two agents who are already matched before they invest and can write complete contracts would make such investments.
} 
the Pareto efficient and individually rational investments for a given pair of agents are characterized by

$$
\delta_{M}^{\prime}\left(\beta_{W}\right) \delta_{W}^{\prime}\left(\beta_{M}\right)=1, \delta_{M}\left(\beta_{W}\right)-\beta_{M} \geq 0 \text { and } \delta_{W}\left(\beta_{M}\right)-\beta_{W} \geq 0
$$

In particular, all these pairs of investments strictly exceed the privately optimal levels $\beta_{M}=0$ and $\beta_{W}=0$.

\subsection{Large Contests with Partially Wasteful or TU Investments}

Theorem 1 and further examination of the matrix $\Theta_{n}$ allow us to obtain precise characterizations of the limit properties of equilibrium utilities, as $n$ and $k$ go to infinity, for matching contests with partially wasteful or TU investments, and to compare these findings with the results for the limit contest with a continuum of agents. For unbalanced markets, we have to assume that matching with a partner who does not invest and has the lowest possible type is not better than staying unmatched, which is formalized here by setting $\underline{w}=0 .{ }^{23}$ To simplify notation, we assume $\underline{w}=0$ also for balanced contests and let $\underline{m}=0$, but these two assumptions do not matter for any of the results.

Condition 1. $\underline{w}=\underline{m}=0$.

\section{The Continuum Model}

We consider here the limit two-sided matching contest with a continuum of agents. The distribution of women is $G$, the distribution of men is $F /(1-r)$ for some $r \in[0,1),{ }^{24}$ and types are private information. Let $m_{r}$ denote the $r$-th quantile of $F$ (i.e., $F\left(m_{r}\right)=$ $r)$, and let $F_{r}(m)=\left(F(m)-F\left(m_{r}\right)\right) /(1-r)$ for $m \geq m_{r}$. The positively assortative matching is now described by the matching function

$$
\psi_{r}(m)= \begin{cases}0 & \text { if } m<m_{r} \\ G^{-1}\left(F_{r}(m)\right) & \text { if } m \geq m_{r}\end{cases}
$$

which is strictly increasing on $\left[m_{r}, \bar{m}\right]$, while types below $m_{r}$ stay unmatched. We let $\phi_{r}$ denote its inverse, defined on $[0, \bar{w}]$.

If all other agents invest according to non-decreasing functions $b_{M}:[0, \bar{m}] \rightarrow \mathbb{R}_{+}$ and $b_{W}:[0, \bar{w}] \rightarrow \mathbb{R}_{+}$that are strictly increasing on $\left[m_{r}, \bar{m}\right]$ and $[0, \bar{w}]$, then a man who

\footnotetext{
${ }^{23}$ If $k$ is large then $E\left[W_{1: k}\right] \approx \underline{w}$, so $\underline{w}=0$ implies that a match with the lowest-ranked woman is not a "large prize" for exogenous reasons (i.e., even if the woman does not invest).

${ }^{24}$ Thus, the total mass of men is $1 /(1-r) \geq 1$.
} 
invests $b_{M}(s)$ knows that he will be matched to a woman with type $\psi_{r}(s)$ who makes an investment $b_{W}\left(\psi_{r}(s)\right)$. Similarly, a woman who invests $b_{W}(s)$ is matched to a man with type $\phi_{r}(s)$ whose investment is $b_{M}\left(\phi_{r}(s)\right)$. Thus, returns to investments are here certain, a significant difference to the finite case.

Equilibrium strategies must clearly satisfy $b_{M}(m)=0$ for $m<m_{r}$ (as these types stay unmatched for sure). Moreover, if $b_{W}(0)=0$ and $b_{M}$ and $b_{W}$ are continuous (as in the equilibrium for the partially wasteful case, Theorem $3 \mathrm{i}$ below), issues related to the question of how to define returns for investments outside of the ranges $b_{M}([0, \bar{m}])$ and $b_{W}([0, \bar{w}])$ do not arise. ${ }^{25}$

Theorem 3. Assume that Condition 1 holds.

i) If $\delta_{M}^{\prime}(0) \delta_{W}^{\prime}(0)<1$, then the continuum model admits a side-symmetric equilibrium $\left(b_{M}, b_{W}\right)$ such that $b_{M}$ and $b_{W}$ are continuous, $b_{W}(0)=0$, and $b_{M}$ and $b_{W}$ are strictly increasing and continuously differentiable on $\left[m_{r}, \bar{m}\right]$ and $[0, \bar{w}]$, respectively. There is a unique equilibrium with these properties. Agents' equilibrium utilities are given by

$$
u_{M}^{(r)}(m)=\gamma_{M} \int_{0}^{m} \psi_{r}(s) d s \text { and } u_{W}^{(r)}(w)=\gamma_{W} \int_{0}^{w} \phi_{r}(s) d s
$$

ii) If $\delta_{M}\left(\beta_{W}\right)=\beta_{W}$ and $\delta_{W}\left(\beta_{M}\right)=\beta_{M}$, the continuum model does not admit an equilibrium that implements the positive assortative matching.

Thus, with partially wasteful investments, assortative matching can arise in equilibrium. With TU investments (case ii), the continuum model does not admit such an equilibrium.

\section{Limit Characterizations}

We now return to our main focus on finite markets. We fix all characteristics of the environment other than the number of agents. That is, $F, G, \gamma_{M}, \gamma_{W}, \delta_{M}$ and $\delta_{W}$ are arbitrary but fixed throughout Section 4.1. We use here superscripts to highlight the dependence of strategies, utilities and expected match partners on $n$ and $k$, writing $b_{M}^{(n, k)}$, $b_{W}^{(n, k)}, a_{M}^{(n, k)}, a_{W}^{(n, k)}, \Psi^{(n, k)}, \Phi^{(n, k)}, U_{M}^{(n, k)}$ and $U_{W}^{(n, k)}$.

We first show that agents' expected utilities in large matching contests with partially wasteful investments converge to the equilibrium utilities for the continuum model:

\footnotetext{
${ }^{25}$ In contrast, the definition of off-equilibrium payoffs plays an important role in continuum models with productive investments, and many different outcomes can potentially be supported as equilibria, depending on the choice of definition. See Peters (2011) for a detailed discussion of this phenomenon. His qualitative results that in large (but finite) unbalanced contests with productive investments, almost all types invest at least weakly (and often strictly) too much compared to (Pareto efficient) hedonic equilibrium investments immediately imply an analogous property for our model with signaling. A thorough analysis of asymptotic behavior in this case is beyond the scope of the present study, however.
} 
Theorem 4. Assume that Condition 1 is satisfied and that $\delta_{M}^{\prime}(0) \delta_{W}^{\prime}(0)<1$. Then, for all $m \in[0, \bar{m}]$ and $w \in[0, \bar{w}]:$

$$
\lim _{k \rightarrow \infty, \frac{k}{n_{k}} \rightarrow 1-r} U_{M}^{\left(n_{k}, k\right)}(m)=u_{M}^{(r)}(m) \text { and } \lim _{k \rightarrow \infty, \frac{k}{n_{k}} \rightarrow 1-r} U_{W}^{\left(n_{k}, k\right)}(w)=u_{W}^{(r)}(w) .
$$

In finite markets, a type's expected utility exceeds his/her information rent $\gamma_{M} \int_{0}^{m} \Psi^{(n, k)}(s) d s$ or $\gamma_{W} \int_{0}^{w} \Phi^{(n, k)}(s) d s$ by a constant equal to the expected benefit from the guaranteed partner's investment, i.e., by $E\left[\delta_{M}\left(b_{W}^{(n, n)}\left(W_{1: n}\right)\right)\right]$ or 0 for men, and by $E\left[\delta_{W}\left(b_{M}^{(n, k)}\left(M_{n-k+1: n}\right)\right)\right]$ for women. ${ }^{26}$ Theorem 4 shows that this extra utility converges to zero if $\delta_{M}^{\prime}(0) \delta_{W}^{\prime}(0)<$ 1 , and also that information rents converge to their continuum counterparts.

Even though signals are only partially wasteful, Theorem 4 implies that, asymptotically, the entire difference between total match surplus

$$
S^{(r)}:=\int_{m_{r}}^{\bar{m}} m \psi_{r}(m) \frac{f(m)}{1-r} d m
$$

and aggregate information rents

$$
\gamma_{M} R_{M}^{(r)}+\gamma_{W} R_{W}^{(r)}
$$

where

$$
R_{M}^{(r)}=\int_{m_{r}}^{\bar{m}}\left(\int_{0}^{m} \psi_{r}(s) d s\right) \frac{f(m)}{1-r} d m \text { and } R_{W}^{(r)}=\int_{0}^{\bar{w}}\left(\int_{0}^{w} \phi_{r}(s) d s\right) g(w) d w
$$

gets dissipated, in accordance with the prediction of Theorem 3 (i). Note that the dissipation rate is always the same, no matter what the benefit functions $\delta_{M}$ and $\delta_{W}$ are (as long as the condition $\delta_{M}^{\prime}(0) \delta_{W}^{\prime}(0)<1$ is satisfied). For example, if $\gamma_{M}=\gamma_{W}=\frac{1}{2}$, exactly half the available surplus is dissipated in the limit, exactly as in the HMS model where signals are completely wasteful and bring no benefit to the other side.

In contrast to these convergence results for partially wasteful investments, the continuum case differs drastically from the finite case under transferable utility. By part (ii) of Theorem 3, the continuum model does not admit an equilibrium since investments grow out of bounds. In the finite model, equilibrium strategies become very steep as $n$ and $k$ grow. Nevertheless, a unique side-symmetric, strictly separating equilibrium always exists (by Theorem 1 and $r(n, k)<1$ ).

We now analyze in more detail the equilibrium of large finite markets with TU

\footnotetext{
${ }^{26}$ Note that the terms $\gamma_{M} \underline{m} E\left[W_{1: n}\right]$ and $\gamma_{W} \underline{w} E\left[M_{n-k+1: n}\right]$ (the type-dependent component of the lowest type's utility from his/her guaranteed match) in (16) and (17) vanish under Condition 1.
} 
investments. This amounts here to understand how the expected investments of the lowest types $M_{1: n}$ and $W_{1: n}$ behave as $n$ goes to infinity.

Note first that ex-post budget balance holds here for each matched pair. In particular, in any balanced market the sum of all agents' ex-ante expected utilities must be equal to the ex-ante expected total match surplus. That is, for all $n$ :

$$
\begin{aligned}
E\left[\frac{1}{n} \sum_{i=1}^{n} M_{i: n} W_{i: n}\right] & =\int_{0}^{\bar{m}} U_{M}^{(n, n)}(m) f(m) d m+\int_{0}^{\bar{w}} U_{W}^{(n, n)}(w) g(w) d w \\
& =E\left[b_{W}^{(n, n)}\left(W_{1: n}\right)\right]+\gamma_{M} \int_{0}^{\bar{m}} \int_{0}^{m} \Psi^{(n, n)}(s) d s f(m) d m \\
& +E\left[b_{M}^{(n, n)}\left(M_{1: n}\right)\right]+\gamma_{W} \int_{0}^{\bar{w}} \int_{0}^{w} \Phi^{(n, n)}(s) d s g(w) d w
\end{aligned}
$$

As $n \rightarrow \infty$, the integral terms converge to $\gamma_{M} R_{M}^{(0)}$ and $\gamma_{W} R_{W}^{(0)}$ (by Lemma 7 in the Appendix and the Dominated Convergence Theorem), and the left hand side converges to $S^{(0)}$ (by the LLN for empirical distributions). Thus,

$$
S^{(0)}=\lim _{n \rightarrow \infty}\left(E\left[b_{M}^{(n, n)}\left(M_{1: n}\right)\right]+E\left[b_{W}^{(n, n)}\left(W_{1: n}\right)\right]\right)+\gamma_{M} R_{M}^{(0)}+\gamma_{W} R_{W}^{(0)}
$$

Because $R_{M}^{(0)}$ and $R_{W}^{(0)}$ are in fact the aggregated core (or stable) payoffs for men and women in the continuum model, we must have

$$
S^{(0)}=R_{M}^{(0)}+R_{W}^{(0)}
$$

Thus, we obtain

$$
\lim _{n \rightarrow \infty}\left(E\left[b_{M}^{(n, n)}\left(M_{1: n}\right)\right]+E\left[b_{W}^{(n, n)}\left(W_{1: n}\right)\right]\right)=\gamma_{W} R_{M}^{(0)}+\gamma_{M} R_{W}^{(0)}>0
$$

We show that the difference between the expected investment made by $M_{1: n}$ and the expected investment made by $W_{1: n}$ converges to 0 , i.e., $\lim _{n \rightarrow \infty}\left(E\left[b_{M}^{(n, n)}\left(M_{1: n}\right)\right]-E\left[b_{W}^{(n, n)}\left(W_{1: n}\right)\right]\right)=$ 0 , leading to our limit characterization of equilibrium utilities in the TU case.

Theorem 5. Assume that Condition 1 is satisfied, and that $\delta_{M}\left(\beta_{W}\right)=\beta_{W}$ and $\delta_{W}\left(\beta_{M}\right)=$ $\beta_{M}$.

i) In large balanced markets, the (per capita) difference between aggregate surplus and aggregate information rents is shared approximately fifty-fifty between men and 
women, regardless of $F, G$ and $\gamma_{M}:$ for all $m \in[0, \bar{m}]$ and $w \in[0, \bar{w}]$,

$$
\begin{aligned}
& \lim _{n \rightarrow \infty} U_{M}^{(n, n)}(m)=\frac{\gamma_{W} R_{M}^{(0)}+\gamma_{M} R_{W}^{(0)}}{2}+\gamma_{M} \int_{0}^{m} \psi_{0}(s) d s, \\
& \lim _{n \rightarrow \infty} U_{W}^{(n, n)}(w)=\frac{\gamma_{W} R_{M}^{(0)}+\gamma_{M} R_{W}^{(0)}}{2}+\gamma_{W} \int_{0}^{w} \phi_{0}(s) d s .
\end{aligned}
$$

ii) In large, unbalanced markets, each agent on the short side obtains a fraction close to 1 of the per capita difference between aggregate surplus and aggregate information rents (on top of her information rent): for all $m \in[0, \bar{m}]$ and $w \in[0, \bar{w}]$,

$$
\begin{aligned}
\lim _{k \rightarrow \infty, k<n_{k}, \frac{k}{n_{k}} \rightarrow 1-r} & U_{M}^{\left(n_{k}, k\right)}(m)=\gamma_{M} \int_{0}^{m} \psi_{r}(s) d s, \\
\lim _{k \rightarrow \infty, k<n_{k}, \frac{k}{n_{k}} \rightarrow 1-r} U_{W}^{\left(n_{k}, k\right)}(w) & =\gamma_{W} R_{M}^{(r)}+\gamma_{M} R_{W}^{(r)}+\gamma_{W} \int_{0}^{w} \phi_{r}(s) d s .
\end{aligned}
$$

Theorem 5 (i) relies on subtle properties of the matrix $\Theta_{n}$. The unbalanced case in Theorem 5 (ii) is much simpler because only agents on the short side obtain utility in excess of information rents, and because only a vanishingly small fraction of total surplus can get dissipated (the investments made by agents failing to match) in large markets.

\subsection{Bounds on Under-Investment when Investments are Productive}

Standard incentives for under-investment (the "hold-up" problem) appear in small matching contests with productive investments (case 3) because the direct benefits from increasing one's investment are realized by the partner. This problem is, at least partly, mitigated by the competition on each side of the market for the external benefits of investments coming from the other side. Moreover, the "exogenous" signaling motive (the competition for partners with higher types) provides additional incentives. Thus, stronger signaling incentives may sometimes help to alleviate hold-up problems, in particular in contests with few participants. In contrast, if the signaling motive is very strong and if Pareto efficient investments are small, or if $n$ and $k$ are very large, signaling will be associated with over-investment in the usual way, driving investments (further) beyond Pareto efficient levels for most pairs of types (compare footnote 25).

We use our sharp condition for equilibrium existence in the linear case (Theorem 1) to derive some interesting bounds on under-investment. Note first that investments $\left(\beta_{M}, \beta_{W}\right)$ with $\delta_{M}^{\prime}\left(\beta_{W}\right) \delta_{W}^{\prime}\left(\beta_{M}\right)>1$ are inefficiently low ${ }^{27}$ while investments

\footnotetext{
${ }^{27}$ There would be a way of marginally increasing both agents' investments that yields a Pareto im-
} 
$\left(\beta_{M}, \beta_{W}\right) \in R_{++}^{2}$ for which $\delta_{M}^{\prime}\left(\beta_{W}\right) \delta_{W}^{\prime}\left(\beta_{M}\right)<1$ correspond to over-investment. Moreover, the level sets $L_{c}:=\left\{\left(\beta_{M}, \beta_{W}\right) \in R_{++}^{2} \mid \delta_{M}^{\prime}\left(\beta_{W}\right) \delta_{W}^{\prime}\left(\beta_{M}\right)=c\right\}$ are closer to $L_{1}$ the closer $c$ is to 1 .

We show first that if $r(n, k) \delta_{M}^{\prime}(0) \delta_{W}^{\prime}(0)<1$, the competition among $n$ men and $k$ women does not suffice to significantly alleviate the hold-up problem if signaling incentives are very small: the multiplier effect due to competition for external benefits is not strong enough for investments to exceed a bounded multiple of those investments that agents would make if signals were completely wasteful. We formalize this insight in Theorem 6, where $n, k, \delta_{M}$ and $\delta_{W}$ are fixed, and where the conditions on the supports of types are necessary and sufficient for signaling incentives to become arbitrarily small independently of the exact form of $F$ and $G .^{28}$

Theorem 6. Assume that $r(n, k) \delta_{M}^{\prime}(0) \delta_{W}^{\prime}(0)<1$. If $n=k, \bar{m}(\bar{w}-\underline{w}) \rightarrow 0$ and $(\bar{m}-$ $\underline{m}) \bar{w} \rightarrow 0$, or if $n>k$ and $\overline{m w} \rightarrow 0$, then $b_{M}(\bar{m}) \rightarrow 0$ and $b_{W}(\bar{w}) \rightarrow 0$ for any sidesymmetric, strictly separating equilibrium.

By contrast, if $r(n, k) \delta_{M}^{\prime}(0) \delta_{W}^{\prime}(0)>1$, the multiplier effect is too strong for investments to get stuck at extremely low levels.

Theorem 7. In any side-symmetric, strictly separating equilibrium, it holds that

$$
r(n, k) \delta_{M}^{\prime}\left(b_{W}(\bar{w})\right) \delta_{W}^{\prime}\left(b_{M}(\bar{m})\right)<1
$$

Thus, if $r(n, k) \delta_{M}^{\prime}(0) \delta_{W}^{\prime}(0)>1$, the hold-up problem is at least partly alleviated by competition, and we obtain a quantitative bound on under-investment. Even with arbitrarily small signaling concerns, it is guaranteed that the investments made by the highest types, $\left(b_{M}(\bar{m}), b_{W}(\bar{w})\right)$, lie above the level set $L_{1 / r(n, k)}$.

\section{Conclusion}

Our paper contributes to the literature that studies bilateral investment behavior in assortative matching markets. We have provided the first quantitative analysis of the multiplier effect shaping Bayes-Nash equilibrium investments in two-sided matching contests when the investments create external benefits, and studied how the effect interacts with agents' signaling incentives. The complex, interdependent nature of equilibrium behavior gives rise to interesting effects: for example, in small markets, raising the sur-

provement for the pair.

${ }^{28}$ Thus, $\Delta_{M}^{a_{M}}$ and $\Delta_{W}^{a_{W}}$ converge to zero. 
plus share of one side of the market may lead to a decrease in expected utility of some agents on that side.

We have identified the spectral radius of a particular matrix of moments of order statistics as the main driver behind the multiplier effect. This characterization has allowed us to shed new light on the relationship between finite and continuum models of matching contests by deriving the exact asymptotic behavior of equilibrium utilities, as the numbers of men and women go to infinity, in environments with partially wasteful or TU investments. Finally, our results also provide novel, quantitative insights into how competition alleviates the hold-up problem in small markets with productive investments.

\section{Appendix}

Proof of Lemma 1. If $b_{W}$ is an equilibrium strategy, it is immediate from (6), (7) and the definitions of $\mathrm{T}$ and $\mathrm{S}$ that $\Delta_{W}^{\delta_{M} \circ b_{W}}$ is a fixed point of $S \circ \hat{T}$. Moreover, the mapping is one-to-one (if $\imath\left(b_{W}^{1}\right)=\imath\left(b_{W}^{2}\right)$ for two equilibrium strategies $b_{W}^{1}$ and $b_{W}^{2}$, then (4) and (5) imply $b_{W}^{1}=b_{W}^{2}$ ) and onto: if $y^{*}$ is a fixed point of $S \circ \hat{T}$, then $b_{M}(m):=\gamma_{M} a_{M}(m)+$ $\hat{\mathscr{F}}(m) \cdot y^{*}$ and $b_{W}(w):=\gamma_{W} a_{W}(w)+\mathscr{G}(w) \cdot \hat{\Delta}_{M}^{\delta_{W} \circ b_{M}}$ constitute equilibrium strategies satisfying $\Delta_{W}^{\delta_{M} \circ b_{W}}=y^{*}$.

Proof of Lemma 2. i) $\theta_{n, i 0}=0$ is obvious. For $j \geq 1$, we first rewrite $\theta_{n, i j}$ using integration by parts: ${ }^{29}$

$$
\begin{aligned}
\theta_{n, i j} & =E\left[F_{j: n-1}\left(M_{i+1: n}\right)-F_{j: n-1}\left(M_{i: n}\right)\right]=\int_{\underline{m}}^{\bar{m}} F_{j: n-1}(m)\left(f_{i+1: n}(m)-f_{i: n}(m)\right) d m \\
& =\int_{\underline{m}}^{\bar{m}}\left(F_{i: n}(m)-F_{i+1: n}(m)\right) f_{j: n-1}(m) d m=E\left[F_{i: n}\left(M_{j: n-1}\right)-F_{i+1: n}\left(M_{j: n-1}\right)\right] .
\end{aligned}
$$

Using the identities (1) and (2), it follows that

$$
\begin{aligned}
\theta_{n, i j} & =E\left[F_{i: n}\left(M_{j: n-1}\right)-F_{i+1: n}\left(M_{j: n-1}\right)\right] \\
& =E\left[\left(\begin{array}{c}
n \\
i
\end{array}\right) F^{i}\left(M_{j: n-1}\right)\left(1-F\left(M_{j: n-1}\right)\right)^{n-i}\right] \\
& =\left(\begin{array}{c}
n \\
i
\end{array}\right)(n-1)\left(\begin{array}{c}
n-2 \\
j-1
\end{array}\right) \int_{\underline{m}}^{\bar{m}} F^{i+j-1}(m)(1-F(m))^{2 n-1-i-j} f(m) d m \\
& =\frac{n-1}{2 n-1} \frac{\left(\begin{array}{c}
n \\
i
\end{array}\right)\left(\begin{array}{c}
n-2 \\
j-1
\end{array}\right)}{\left(\begin{array}{c}
2 n-2 \\
i+j-1
\end{array}\right)} \int_{\underline{m}}^{\bar{m}} f_{i+j: 2 n-1}(m) d m=\frac{n-1}{2 n-1} \frac{\left(\begin{array}{c}
n \\
i
\end{array}\right)\left(\begin{array}{c}
n-2 \\
j-1
\end{array}\right)}{\left(\begin{array}{c}
2 n-2 \\
i+j-1
\end{array}\right)} .
\end{aligned}
$$

\footnotetext{
${ }^{29}$ Observe that the case $i=0$ (so that $F_{0: n} \equiv 1$ on $[\underline{m}, \bar{m}]$ ) is covered by the argument.
} 
ii) Case $n=2$ : The formula from (i) yields $\theta_{2,01}=\theta_{2,11}=\frac{1}{3}$. Thus, $3 \Theta_{2}$ is stochastic.

Case $n>2$ : The entries of the i-th row of $\Theta_{n}$ can be reinterpreted in a way that allows computing the row sum as a telescoping sum. Indeed, for $j=2, \ldots, n-2$ :

$$
\begin{aligned}
E & {\left[F_{j-1: n-2}\left(M_{i+1: n+1}\right)-F_{j: n-2}\left(M_{i+1: n+1}\right)\right] } \\
& =\int_{\underline{m}}^{\bar{m}}\left(\begin{array}{c}
n-2 \\
j-1
\end{array}\right) F^{j-1}(m)(1-F(m))^{n-1-j} f_{i+1: n+1}(m) d m \\
& =\left(\begin{array}{c}
n-2 \\
j-1
\end{array}\right)(n+1)\left(\begin{array}{c}
n \\
i
\end{array}\right) \int_{\underline{m}}^{\bar{m}} F^{i+j-1}(m)(1-F(m))^{2 n-1-i-j} f(m) d m \\
& =\frac{n+1}{2 n-1} \frac{\left(\begin{array}{c}
n \\
i
\end{array}\right)\left(\begin{array}{c}
n-2 \\
j-1
\end{array}\right)}{\left(\begin{array}{c}
2 n-2 \\
i+j-1
\end{array}\right)}=\frac{n+1}{n-1} \theta_{n, i j} .
\end{aligned}
$$

Similarly, for $j=n-1$ we find:

$$
\begin{aligned}
E & {\left[F_{n-2: n-2}\left(M_{i+1: n+1}\right)\right]=\int_{\underline{m}}^{\bar{m}}\left(\begin{array}{l}
n-2 \\
n-2
\end{array}\right) F^{n-2}(m) f_{i+1: n+1}(m) d m } \\
& =\left(\begin{array}{l}
n-2 \\
n-2
\end{array}\right)(n+1)\left(\begin{array}{c}
n \\
i
\end{array}\right) \int_{\underline{m}}^{\bar{m}} F^{i+(n-1)-1}(m)(1-F(m))^{2 n-1-i-(n-1)} f(m) d m \\
& =\frac{n+1}{2 n-1} \frac{\left(\begin{array}{c}
n \\
i
\end{array}\right)\left(\begin{array}{c}
n-2 \\
n-2
\end{array}\right)}{\left(\begin{array}{c}
2 n-2 \\
i+(n-1)-1
\end{array}\right)}=\frac{n+1}{n-1} \theta_{n, i(n-1)} .
\end{aligned}
$$

Finally, for $j=1$,

$$
E\left[1-F_{1: n-2}\left(M_{i+1: n+1}\right)\right]=\frac{n+1}{n-1} \theta_{n, i 1} .
$$

Summing up all terms (including $\theta_{n, i 0}=0$ ) yields $\frac{n+1}{n-1} \sum_{j=0}^{n-1} \theta_{n, i j}=1$.

Proof of Theorem 1. If $d_{M}=0$, the unique fixed point of $S \circ \hat{T}$ is 0 , and (11) and (12) follow immediately from (9) and (10). If $d_{M}>0$, the fixed point equation becomes

$$
\Delta_{W}^{b_{W}}=\gamma_{W} \Delta_{W}^{a_{W}}+d_{W} \Theta_{k}\left(\gamma_{M} \hat{\Delta}_{M}^{a_{M}}+d_{M} \hat{\Theta}_{n, k} \Delta_{W}^{b_{W}}\right)
$$

or

$$
\left(I_{k}-d_{M} d_{W} \Theta_{k} \hat{\Theta}_{n, k}\right) \Delta_{W}^{b_{W}}=\gamma_{W} \Delta_{W}^{a_{W}}+d_{W} \gamma_{M} \Theta_{k} \hat{\Delta}_{M}^{a_{M}} \cdot 30
$$

Case $r(n, k) d_{M} d_{W}<1: \quad r(n, k) d_{M} d_{W}$ is the spectral radius of $d_{M} d_{W} \Theta_{k} \hat{\Theta}_{n, k}$. It follows that $I_{k}-d_{M} d_{W} \Theta_{k} \hat{\Theta}_{n, k}$ is invertible, so that (18) yields (12). We must also show $\Delta_{W}^{b_{W}} \geq 0$. As $r(n, k) d_{M} d_{W}<1,\left(I_{k}-d_{M} d_{W} \Theta_{k} \hat{\Theta}_{n, k}\right)^{-1}$ is given by the convergent series $\sum_{l=0}^{\infty}\left(d_{M} d_{W} \Theta_{k} \hat{\Theta}_{n, k}\right)^{l}$. Thus, $\left(I_{k}-d_{M} d_{W} \Theta_{k} \hat{\Theta}_{n, k}\right)^{-1} \geq 0$. Moreover, $\Delta_{W}^{a_{W}}>0$ because $a_{W}$

\footnotetext{
${ }^{30}$ We have derived the equation directly from (9) and (10). This is equivalent to writing down the fixed point equation $S \circ \hat{T}(y)=y$ for $y=d_{M} \Delta_{W}^{b_{W}}$ and then dividing this equation by the constant $d_{M}>0$.
} 
is strictly increasing (for $j \geq 1, w \mathscr{G}_{j}(w)-\int_{w}^{w} \mathscr{G}_{j}(s) d s$ is strictly increasing, and $\hat{\Delta}_{M}^{I_{1}}>0$ ). Similarly, $\hat{\Delta}_{M}^{a_{M}}>0$. As $\Theta_{k} \geq 0$, it follows that $\gamma_{W} \Delta_{W}^{a_{W}}+d_{W} \gamma_{M} \Theta_{k} \hat{\Delta}_{M}^{a_{M}}>0$. Thus, $\Delta_{W}^{b_{W}} \geq 0$ (in fact, $\Delta_{W}^{b_{W}}>0$, because the diagonal entries of $\left(I_{k}-d_{M} d_{W} \Theta_{k} \hat{\Theta}_{n, k}\right)^{-1}$ are positive).

Finally, the explicit form of $\hat{\Delta}_{M}^{b_{M}}$, stated in (11), follows from plugging (10) into (9).

Case $r(n, k) d_{M} d_{W} \geq 1$ : let $z:=\gamma_{W} \Delta_{W}^{a_{W}}+d_{W} \gamma_{M} \Theta_{k} \hat{\Delta}_{M}^{a_{M}}$. We have already shown that $z>0$. We have to show that the linear system of equations $\left(I_{k}-d_{M} d_{W} \Theta_{k} \hat{\Theta}_{n, k}\right) v=z$ has no solution $v \in \mathbb{R}_{+}^{k}$. This follows from Farkas' Lemma and from the fact that the non-negative and non-zero matrix $\Theta_{k} \hat{\Theta}_{n, k}$, has a non-negative, non-zero left eigenvector $w(n, k)$ associated with $r(n, k)$, i.e., $w(n, k)^{T} \Theta_{k} \hat{\Theta}_{n, k}=r(n, k) w(n, k)^{T}$ (see Theorem 8.3.1 in Horn and Johnson 2013). ${ }^{31}$ It follows that

$$
w(n, k)^{T}\left(I_{k}-d_{M} d_{W} \Theta_{k} \hat{\Theta}_{n, k}\right)=w(n, k)^{T}\left(1-r(n, k) d_{M} d_{W}\right) \leq 0 \text { and } w(n, k)^{T} z>0 .
$$

Thus, by Farkas' Lemma, $\left(I_{k}-d_{M} d_{W} \Theta_{k} \hat{\Theta}_{n, k}\right) v=z$ has no solution $v \geq 0$, which concludes the proof for the case $r(n, k) d_{M} d_{W} \geq 1$.

Proof of Theorem 2. We must show that $S \circ \hat{T}$ has a fixed point. Recall that

$\hat{T}_{i}(y)=E\left[\delta_{W}\left(\gamma_{M} a_{M}\left(M_{n-k+i+1: n}\right)+\hat{\mathscr{F}}\left(M_{n-k+i+1: n}\right) \cdot y\right)-\delta_{W}\left(\gamma_{M} a_{M}\left(M_{n-k+i: n}\right)+\hat{\mathscr{F}}\left(M_{n-k+i: n}\right) \cdot y\right)\right]$,

and

$$
S_{i}(x)=E\left[\delta_{M}\left(\gamma_{W} a_{W}\left(W_{i+1: k}\right)+\mathscr{G}\left(W_{i+1: k}\right) \cdot x\right)-\delta_{M}\left(\gamma_{W} a_{W}\left(W_{i: k}\right)+\mathscr{G}\left(W_{i: k}\right) \cdot x\right)\right],
$$

for $i \in\{0, \ldots, k-1\}$. We prove the theorem for $n>k$ first. In this case $\hat{\mathscr{F}}_{0}=\mathscr{F}_{n-k} \not \equiv 0$, so that $\hat{T}(y)$ depends on all entries of $y$. $\hat{T}_{0}(y)$ has no effect on $S(\hat{T}(y))$ because $\mathscr{G}_{0} \equiv 0$. Given a vector $v=\left(v_{0}, \ldots, v_{k-1}\right) \in \mathbb{R}^{k}$, we write $v_{-0}$ for the vector $\left(v_{1}, \ldots, v_{k-1}\right)$.

By assumption, $\frac{(n-1)(k-1)}{(n+1)(k+1)}\left(\lim _{b \rightarrow \infty} \delta_{M}^{\prime}(b)\right)\left(\lim _{b \rightarrow \infty} \delta_{W}^{\prime}(b)\right)<1$. We pick two constants, $C_{M}$ and $C_{W}$, such that $\lim _{b \rightarrow \infty} \delta_{M}^{\prime}(b)<C_{M}, \lim _{b \rightarrow \infty} \delta_{W}^{\prime}(b)<C_{W}$ and $\frac{(n-1)(k-1)}{(n+1)(k+1)} C_{W} C_{M}<$ 1. Then, there are constants $0<b^{1}<b^{2}<\infty$ such that i) for all $b \geq b^{1}, \delta_{M}^{\prime}(b) \leq C_{M}$ and $\delta_{W}^{\prime}(b) \leq C_{W}$, and ii) for all $b \geq b^{2}, \delta_{M}(b) \leq C_{M} b$.

For each $y \in \mathbb{R}_{+}^{k}, \gamma_{M} a_{M}\left(M_{n-k+1: n}\right)+\hat{\mathscr{F}}\left(M_{n-k+1: n}\right) \cdot y$ is the smallest one of the terms $\gamma_{M} a_{M}\left(M_{n-k+i: n}\right)+\hat{\mathscr{F}}\left(M_{n-k+i: n}\right) \cdot y, i \in\{1, \ldots, k\}$, that occur in the definition of $\hat{T}_{-0}$. Moreover, the smallest entry of $\hat{\mathscr{F}}\left(M_{n-k+1: n}\right)=\left(F_{n-k+j: n-1}\left(M_{n-k+1: n}\right)\right)_{j=0, \ldots, k-1}$

\footnotetext{
${ }^{31}$ In fact, Lemma 2 implies that for $n>k, \Theta_{k} \hat{\Theta}_{n, k}>0$, so that $w(n, k)>0$ by Perron's Theorem (Theorem 8.2.8 in Horn and Johnson 2013). For $n=k$, the first column of $\Theta_{k} \hat{\Theta}_{n, k}=\Theta_{n}^{2}$ is zero, and the $(n-1) \times(n-1)$-submatrix that results from deleting the first row and the first column is positive. Thus, by Perron's Theorem (applied to the submatrix), $w(n, n)_{0}=0$ and $w(n, n)_{i}>0$ for $i \in\{1, \ldots, n-1\}$.
} 
is $F_{n-1: n-1}\left(M_{n-k+1: n}\right)$, because $F_{n-1: n-1}$ first-order stochastically dominates the distributions of the lower order statistics. This implies $\gamma_{M} a_{M}\left(M_{n-k+1: n}\right)+\hat{\mathscr{F}}\left(M_{n-k+1: n}\right) \cdot y \geq$ $\|y\|_{\infty} F_{n-1: n-1}\left(M_{n-k+1: n}\right)$. For $b \geq b^{1}$, we define $m(b)$ via $b F_{n-1: n-1}(m(b))=b^{1} . m(b)$ is decreasing in $b$ and converges to $\underline{m}$ as $b \rightarrow \infty$. We split every $\hat{T}_{i}(y), i \in\{1, \ldots, k-1\}$, into two terms: $\hat{T}_{i}(y)=A_{i}^{\hat{T}}(y)+\varepsilon_{i}^{\hat{T}}(y)$, where

$$
\begin{aligned}
A_{i}^{\hat{T}}(y) & =E\left[\left(\delta_{W}\left(\gamma_{M} a_{M}\left(M_{n-k+i+1: n}\right)+\hat{\mathscr{F}}\left(M_{n-k+i+1: n}\right) \cdot y\right)\right.\right. \\
& \left.\left.-\delta_{W}\left(\gamma_{M} a_{M}\left(M_{n-k+i: n}\right)+\hat{\mathscr{F}}\left(M_{n-k+i: n}\right) \cdot y\right)\right) I_{\left\{M_{n-k+1: n} \geq m\left(\|y\|_{\infty}\right)\right\}}\right]
\end{aligned}
$$

and

$$
\begin{aligned}
\varepsilon_{i}^{\hat{T}}(y) & =E\left[\left(\delta_{W}\left(\gamma_{M} a_{M}\left(M_{n-k+i+1: n}\right)+\hat{\mathscr{F}}\left(M_{n-k+i+1: n}\right) \cdot y\right)\right.\right. \\
& \left.\left.-\delta_{W}\left(\gamma_{M} a_{M}\left(M_{n-k+i: n}\right)+\hat{\mathscr{F}}\left(M_{n-k+i: n}\right) \cdot y\right)\right) I_{\left\{M_{n-k+1: n}<m\left(\|y\|_{\infty}\right)\right\}}\right]
\end{aligned}
$$

where $I_{\{\cdot\}}$ is an indicator function. We use the mean value theorem and the bound $\delta_{W}^{\prime}(b) \leq C_{W}$ for $b>b^{1}$ to obtain an upper bound for $A_{i}^{\hat{T}}(y)$ :

$$
\begin{aligned}
A_{i}^{\hat{T}}(y) & \leq C_{W} E\left[\left(\gamma_{M}\left(a_{M}\left(M_{n-k+i+1: n}\right)-a_{M}\left(M_{n-k+i: n}\right)\right)\right.\right. \\
& \left.\left.+\left(\hat{\mathscr{F}}\left(M_{n-k+i+1: n}\right)-\hat{\mathscr{F}}\left(M_{n-k+i: n}\right)\right) \cdot y\right) I_{\left\{M_{n-k+1: n} \geq m\left(|y|_{\infty}\right)\right\}}\right] \\
& \leq C_{W}\left(\gamma_{M} \hat{\Delta}_{M, i}^{a_{M}}+\sum_{j=0}^{k-1} E\left[F_{n-k+j: n-1}\left(M_{n-k+i+1: n}\right)-F_{n-k+j: n-1}\left(M_{n-k+i: n}\right)\right] y_{j}\right) \\
& =C_{W}\left(\gamma_{M} \hat{\Delta}_{M, i}^{a_{M}}+\left(\hat{\Theta}_{n, k} y\right)_{i}\right)
\end{aligned}
$$

The terms $\varepsilon_{i}^{\hat{T}}(y)$ are of order $o\left(\|y\|_{\infty}\right)$ for $\|y\|_{\infty} \rightarrow \infty$. Indeed, $\varepsilon_{i}^{\hat{T}}(y)>0$ and

$$
\begin{aligned}
\sum_{i=1}^{k-1} \varepsilon_{i}^{\hat{T}}(y) & \leq E\left[\left(\delta_{W}\left(\gamma_{M} a_{M}\left(M_{n: n}\right)+\hat{\mathscr{F}}\left(M_{n: n}\right) \cdot y\right)\right) I_{\left\{M_{n-k+1: n}<m\left(\|y\|_{\infty}\right)\right\}}\right] \\
& \leq \delta_{W}\left(\gamma_{M} a_{M}(\bar{m})+k\|y\|_{\infty}\right) E\left[I_{\left\{M_{n-k+1: n}<m\left(\|y\|_{\infty}\right)\right\}}\right]
\end{aligned}
$$

As $\|y\|_{\infty} \rightarrow \infty$, the first factor is of order $O\left(\|y\|_{\infty}\right)$, and the second is of order $o(1)$. Thus, their product is of order $o\left(\|y\|_{\infty}\right)$. Combining the estimates, we get

$$
\hat{T}_{i}(y) \leq C_{W}\left(\hat{\Theta}_{n, k} y\right)_{i}+o\left(\|y\|_{\infty}\right)
$$


In particular,

$$
\left\|\hat{T}_{-0}(y)\right\|_{\infty} \leq C_{W} \frac{n-1}{n+1}\|y\|_{\infty}+o\left(\|y\|_{\infty}\right)
$$

because $\frac{n+1}{n-1} \Theta_{n}$ is a stochastic matrix. Consider $S$ then. $\gamma_{W} a_{W}\left(W_{1: k}\right)+\mathscr{G}\left(W_{1: k}\right) \cdot x$ is the smallest non-zero term among the terms $\gamma_{W} a_{W}\left(W_{i: k}\right)+\mathscr{G}\left(W_{i: k}\right) \cdot x=\gamma_{W} a_{W}\left(W_{i: k}\right)+$ $\mathscr{G}_{-0}\left(W_{i: k}\right) \cdot x_{-0}, i \in\{0, \ldots, k\}$, that occur in the definition of $S$ (and $\gamma_{W} a_{W}\left(W_{0: k}\right)+\mathscr{G}\left(W_{0: k}\right)$. $x=0)$. Moreover, $\gamma_{W} a_{W}\left(W_{1: k}\right)+\mathscr{G}_{-0}\left(W_{1: k}\right) \cdot x_{-0} \geq\left\|x_{-0}\right\|_{\infty} G_{k-1: k-1}\left(W_{1: k}\right)$. For $l \in$ $\{1,2\}$, and for $b \geq b^{l}$, we define $w^{l}(b)$ via $b G_{k-1: k-1}\left(w^{l}(b)\right)=b^{l}$.

We discuss the terms $S_{i}(x)$ for $i \geq 1$ first. We write $S_{i}(x)=A_{i}^{S}(x)+\varepsilon_{i}^{S}(x)$, where

$$
\begin{aligned}
A_{i}^{S}(x) & =E\left[\left(\delta_{M}\left(\gamma_{W} a_{W}\left(W_{i+1: k}\right)+\mathscr{G}_{-0}\left(W_{i+1: k}\right) \cdot x_{-0}\right)\right.\right. \\
& \left.\left.-\delta_{M}\left(\gamma_{W} a_{W}\left(W_{i: k}\right)+\mathscr{G}_{-0}\left(W_{i: k}\right) \cdot x_{-0}\right)\right) I_{\left\{W_{1: k} \geq w^{1}\left(\left\|x_{-0}\right\|_{\infty}\right)\right\}}\right]
\end{aligned}
$$

and

$$
\begin{aligned}
\varepsilon_{i}^{S}(x) & =E\left[\left(\delta_{M}\left(\gamma_{W} a_{W}\left(W_{i+1: k}\right)+\mathscr{G}_{-0}\left(W_{i+1: k}\right) \cdot x_{-0}\right)\right.\right. \\
& \left.\left.-\delta_{M}\left(\gamma_{W} a_{W}\left(W_{i: k}\right)+\mathscr{G}_{-0}\left(W_{i: k}\right) \cdot x_{-0}\right)\right) I_{\left\{W_{1: k}<w^{1}\left(\left\|x_{-0}\right\|_{\infty}\right)\right\}}\right]
\end{aligned}
$$

Analogously to the estimates for $\hat{T}$, it follows that

$$
\begin{aligned}
A_{i}^{S}(x) & \leq C_{M}\left(\gamma_{W} \Delta_{W, i}^{a_{W}}+\sum_{j=1}^{k-1} E\left[G_{j: k-1}\left(W_{i+1: k}\right)-G_{j: k-1}\left(W_{i: k}\right)\right] x_{j}\right) \\
& =C_{M}\left(\gamma_{W} \Delta_{W, i}^{a_{W}}+\sum_{j=1}^{k-1} \theta_{k, i j} x_{j}\right)
\end{aligned}
$$

and $\varepsilon_{i}^{S}(x)=o\left(\left\|x_{-0}\right\|_{\infty}\right)$. Thus, $S_{i}(x) \leq C_{M} \sum_{j=1}^{k-1} \theta_{k, i j} x_{j}+o\left(\left\|x_{-0}\right\|_{\infty}\right)$ for $i \geq 1$. We then write $S_{0}(x)$, using $w^{2}$, as $S_{0}(x)=A_{0}^{S}(x)+\varepsilon_{0}^{S}(x)$, where

$$
A_{0}^{S}(x)=E\left[\left(\delta_{M}\left(\gamma_{W} a_{W}\left(W_{1: k}\right)+\mathscr{G}_{-0}\left(W_{1: k}\right) \cdot x_{-0}\right)\right) I_{\left\{W_{1: k} \geq w^{2}\left(\left\|x_{-0}\right\|_{\infty}\right)\right\}}\right]
$$

and

$$
\varepsilon_{0}^{S}(x)=E\left[\left(\delta_{M}\left(\gamma_{W} a_{W}\left(W_{1: k}\right)+\mathscr{G}_{-0}\left(W_{1: k}\right) \cdot x_{-0}\right)\right) I_{\left\{W_{1: k}<w^{2}\left(\left\|x_{-0}\right\|_{\infty}\right)\right\}}\right] .
$$

Then $\varepsilon_{0}^{S}(x)=o\left(\left\|x_{-0}\right\|_{\infty}\right)$, and $A_{0}^{S}(x) \leq C_{M}\left(\gamma_{W} \Delta_{W, 0}^{a_{W}}+\sum_{j=1}^{k-1} \theta_{k, 0 j} x_{j}\right)$. Combining all estimates for $S$, and using that $\frac{k+1}{k-1} \Theta_{k}$ is stochastic, we get

$$
\|S(x)\|_{\infty} \leq C_{M} \frac{k-1}{k+1}\left\|x_{-0}\right\|_{\infty}+o\left(\left\|x_{-0}\right\|_{\infty}\right),
$$


and thus

$$
\begin{aligned}
\|S(\hat{T}(y))\|_{\infty} & \leq C_{M} \frac{k-1}{k+1}\left\|\hat{T}_{-0}(y)\right\|_{\infty}+o\left(\left\|\hat{T}_{-0}(y)\right\|_{\infty}\right) \\
& \leq C_{M} C_{W} \frac{(k-1)(n-1)}{(k+1)(n+1)}\|y\|_{\infty}+o\left(\|y\|_{\infty}\right) .
\end{aligned}
$$

As $C_{M} C_{W} \frac{(k-1)(n-1)}{(k+1)(n+1)}<1$, it follows that there is some $K_{1}<\infty$ such that for all $y \in \mathbb{R}_{+}^{k}$ with $\|y\|_{\infty} \geq K_{1},\|S(\hat{T}(y))\|_{\infty} \leq\|y\|_{\infty}$. For any $K>0$, let $\bar{B}_{K}(0)$ be the closure of the ball of radius $K$ in $\mathbb{R}^{k}$ with respect to $\|\cdot\|_{\infty} . \hat{T}\left(\bar{B}_{K_{1}}(0) \cap \mathbb{R}_{+}^{k}\right)$ is compact $\left(\bar{B}_{K_{1}}(0) \cap \mathbb{R}_{+}^{k}\right.$ is compact and $\hat{T}$ is continuous). Taken together, the two observations imply that there is some $K_{2}$ (possibly greater than $\left.K_{1}\right)$, such that $S\left(\hat{T}\left(\bar{B}_{K_{2}}(0) \cap \mathbb{R}_{+}^{k}\right)\right) \subset \bar{B}_{K_{2}}(0) \cap \mathbb{R}_{+}^{k}$. Thus, the continuous mapping $S \circ \hat{T}$ maps the compact and convex set $\bar{B}_{K_{2}}(0) \cap \mathbb{R}_{+}^{k}$ into itself. By Brouwer's Theorem, $S \circ \hat{T}$ has a fixed point, which concludes the proof for the case $n>k$.

The proof for $n=k$ is analogous. In this case, both $y_{0}$ (corresponding to $\Delta_{W, 0}^{\delta_{M} \circ b_{W}}$ ) and $x_{0}$ (corresponding to $\hat{\Delta}_{M, 0}^{\delta_{W} \circ b_{M}}$ ) are irrelevant. Hence, it suffices to show that $S_{-0} \circ \hat{T}_{-0}$ : $\mathbb{R}_{+}^{k-1} \rightarrow \mathbb{R}_{+}^{k-1}$ has a fixed point, and the arguments to obtain the estimates for applying Brouwer's Theorem use only the bounds $\delta_{M}^{\prime}(b) \leq C_{M}$ and $\delta_{W}^{\prime}(b) \leq C_{W}$ for $b \geq b^{1}$.

Lemma 4. $\Delta_{W}^{a_{W}}$ and $\Delta_{M}^{a_{M}}$ satisfy

$$
\begin{aligned}
\Delta_{W, i}^{a_{W}} & =\sum_{j=1}^{k-1} \theta_{k, i j} E\left[W_{i+j: 2 k-1}\right] \hat{\Delta}_{M, j}^{I_{1}} & & \text { for } i \in\{0, \ldots, k-1\}, \\
\Delta_{M, i}^{a_{M}} & =\sum_{j=0}^{k-1} \theta_{n, i(n-k+j)} E\left[M_{i+n-k+j: 2 n-1}\right] \Delta_{W, j}^{I_{1}} & & \text { for } i \in\{0, \ldots, n-1\} .
\end{aligned}
$$

Proof of Lemma 4. i) Consider $i \geq 1$ first. Using $w G_{j: k-1}(w)-\int_{\underline{w}}^{w} G_{j: k-1}(s) d s=\int_{\underline{w}}^{w} s g_{j: k-1}(s) d s$ 
for $j \geq 1$, we find:

$$
\begin{aligned}
\Delta_{W, i}^{a_{W}}= & E\left[a_{W}\left(W_{i+1: k}\right)-a_{W}\left(W_{i: k}\right)\right] \\
= & \sum_{j=1}^{k-1} E\left[\int_{\underline{w}}^{W_{i+1: k}} s g_{j: k-1}(s) d s-\int_{\underline{w}}^{W_{i: k}} s g_{j: k-1}(s) d s\right] \hat{\Delta}_{M, j}^{I_{1}} \\
= & \sum_{j=1}^{k-1}\left(\int_{\underline{w}}^{\bar{w}}\left(\int_{\underline{w}}^{w} s g_{j: k-1}(s) d s\right)\left(g_{i+1: k}(w)-g_{i: k}(w)\right) d w\right) \hat{\Delta}_{M, j}^{I_{1}} \\
= & \sum_{j=1}^{k-1}\left(\int_{\underline{w}}^{\bar{w}} w g_{j: k-1}(w)\left(G_{i: k}(w)-G_{i+1: k}(w)\right) d w\right) \hat{\Delta}_{M, j}^{I_{1}} \\
= & \sum_{j=1}^{k-1}\left(\int_{\underline{w}}^{\bar{w}} w(k-1)\left(\begin{array}{c}
k-2 \\
j-1
\end{array}\right) G(w)^{j-1}(1-G(w))^{k-1-j} g(w)\left(\begin{array}{c}
k \\
i
\end{array}\right) G(w)^{i}(1-G(w))^{k-i} d w\right) \hat{\Delta}_{M, j}^{I_{1}} \\
= & \sum_{j=1}^{k-1} \theta_{k, i j}\left(\int_{\underline{w}}^{\bar{w}} w(2 k-1)\left(\begin{array}{c}
2 k-2 \\
i+j-1
\end{array}\right) G(w)^{i+j-1}(1-G(w))^{2 k-1-(i+j)} g(w) d w\right) \hat{\Delta}_{M, j}^{I_{1}} \\
= & \sum_{j=1}^{k-1} \theta_{k, i j} E\left[W_{i+j: 2 k-1}\right] \hat{\Delta}_{M, j}^{I_{1}} .
\end{aligned}
$$

Similarly, for $\Delta_{M, i}^{a_{M}}, i \geq 1$ (if $n=k$, the term for $j=0$ in the following calculation is trivially equal to zero):

$$
\begin{aligned}
\Delta_{M, i}^{a_{M}} & =E\left[a_{M}\left(M_{i+1: n}\right)-a_{M}\left(M_{i: n}\right)\right] \\
& =\sum_{j=0}^{k-1} E\left[\int_{\underline{m}}^{M_{i+1: n}} s f_{n-k+j: n-1}(s) d s-\int_{\underline{m}}^{M_{i: n}} s f_{n-k+j: n-1}(s) d s\right] \Delta_{W, j}^{I_{1}} \\
& =\sum_{j=0}^{k-1}\left(\int_{\underline{m}}^{\bar{m}}\left(\int_{\underline{m}}^{m} s f_{n-k+j: n-1}(s) d s\right)\left(f_{i+1: n}(m)-f_{i: n}(m)\right) d m\right) \Delta_{W, j}^{I_{1}} \\
& =\sum_{j=0}^{k-1}\left(\int_{\underline{m}}^{\bar{m}} m f_{n-k+j: n-1}(m)\left(F_{i: n}(m)-F_{i+1: n}(m)\right) d m\right) \Delta_{W, j}^{I_{1}} \\
& =\sum_{j=0}^{k-1} \theta_{n, i(n-k+j)} E\left[M_{i+n-k+j: 2 n-1}\right] \Delta_{W, j}^{I_{1}} .
\end{aligned}
$$

The proofs for $i=0$ follow from analogous calculations (using $a_{W}\left(W_{0: k}\right)=a_{M}\left(M_{0: n}\right)=$ 0 and $\left.1-G_{1: k}(\bar{w})=1-F_{1: n}(\bar{m})=0\right)$.

In Section 3.1, we need the following fact about order statistics of distributions with increasing virtual valuations.

Lemma 5. Suppose that $F$ has increasing virtual valuations, i.e., $m-\frac{1-F(m)}{f(m)}$ is weakly 
increasing. Then the following inequality holds:

$$
4 E\left[M_{1: 3}\right] \geq E\left[M_{2: 3}\right]
$$

(19) is satisfied with equality if virtual valuations are constant.

Proof. Recall that $F_{1: 3}=1-(1-F)^{3}$ and $F_{1: 3}-F_{2: 3}=3 F(1-F)^{2}$. We can thus write

$$
\begin{aligned}
4 E\left[M_{1: 3}\right]-E\left[M_{2: 3}\right] & =3 E\left[M_{1: 3}\right]+\left(E\left[M_{1: 3}\right]-E\left[M_{2: 3}\right]\right) \\
& =3 \int_{0}^{\bar{m}}(1-F(x))^{3} d x-3 \int_{0}^{\bar{m}} F(x)(1-F(x))^{2} d x \\
& =3 \int_{0}^{\bar{m}}(1-F(x))^{2}(1-2 F(x)) d x \\
& =3\left(\int_{0}^{\bar{m}}(1-F(x))^{2} d x-\int_{0}^{\bar{m}}(1-F(x))^{2} 2 F(x) d x\right) .
\end{aligned}
$$

Defining $h=(1-F)^{2} / f$, this gives us

$$
\begin{aligned}
4 E\left[M_{1: 3}\right]-E\left[M_{2: 3}\right] & =3 \int_{0}^{\bar{m}} h(x) f(x) d x-\int_{0}^{\bar{m}} h(x) 2 F(x) f(x) d x \\
& =3 E[h(M)]-3 E\left[h\left(M_{2: 2}\right)\right]
\end{aligned}
$$

where the random variable $M$ is distributed according to $F$ and where we used that $f_{2: 2}=2 F f$. Thus, if $h$ is decreasing, $4 E\left[M_{1: 3}\right]-E\left[M_{2: 3}\right]$ is non-negative. Yet this is indeed the case since $1 / h$ is the so-called zoom rate associated with the distribution $F$ and since it is shown in Ewerhart (2013) that the zoom rate being increasing is equivalent to our assumption of increasing virtual valuations, see also Szech (2011).

Proof of Lemma 3. We give the proof for $U_{M}$ and $n=k$. The remaining cases are analogous. Invoking (4) and the formula for $a_{M}$, we find:

$$
\begin{aligned}
U_{M}(m) & =\gamma_{M} m \Psi(m)+E\left[\delta_{M}\left(b_{W}\left(W_{1: n}\right)\right)\right]+\hat{\mathscr{F}}(m) \cdot \Delta_{W}^{\delta_{M} \circ b_{W}}-b_{M}(m) \\
& =\gamma_{M} m\left(E\left[W_{1: n}\right]+\hat{\mathscr{F}}(m) \cdot \Delta_{W}^{I_{1}}\right)+E\left[\delta_{M}\left(b_{W}\left(W_{1: n}\right)\right)\right]-\gamma_{M} a_{M}(m) \\
& =E\left[\delta_{M}\left(b_{W}\left(W_{1: n}\right)\right)\right]+\gamma_{M}\left(m E\left[W_{1: n}\right]+\int_{\underline{m}}^{m} \hat{\mathscr{F}}(s) d s \cdot \Delta_{W}^{I_{1}}\right) \\
& =E\left[\delta_{M}\left(b_{W}\left(W_{1: n}\right)\right)\right]+\gamma_{M} \underline{m} E\left[W_{1: n}\right]+\gamma_{M} \int_{\underline{m}}^{m} \Psi(s) d s .
\end{aligned}
$$

Proof of Theorem 3. Assuming that others invest according to continuous, non-decreasing functions $b_{M}$ and $b_{W}$ that are strictly increasing and differentiable on $\left[m_{r}, \bar{m}\right]$ and $[\underline{w}, \bar{w}]$ 
and satisfy $b_{M}\left(m_{r}\right)=b_{W}(0)=0$, the maximization problems for types $m$ and $w$ are:

$$
\begin{aligned}
& \max _{s \in[0, \bar{m}]}\left[\gamma_{M} m \psi_{r}(s)-b_{M}(s)+\delta_{M}\left(b_{W}\left(\psi_{r}(s)\right)\right)\right], \text { and } \\
& \max _{s \in[0, \bar{w}]}\left[\gamma_{W} w \phi_{r}(s)-b_{W}(s)+\delta_{W}\left(b_{M}\left(\phi_{r}(s)\right)\right)\right] .
\end{aligned}
$$

This implies the following necessary conditions for equilibrium investments by types $m>m_{r}$ and $w>0$ :

$$
\begin{aligned}
& b_{M}^{\prime}(m)=\gamma_{M} m \psi_{r}^{\prime}(m)+\delta_{M}^{\prime}\left(b_{W}\left(\psi_{r}(m)\right)\right) b_{W}^{\prime}\left(\psi_{r}(m)\right) \psi_{r}^{\prime}(m) \\
& b_{W}^{\prime}(w)=\gamma_{W} w \phi_{r}^{\prime}(w)+\delta_{W}^{\prime}\left(b_{M}\left(\phi_{r}(w)\right)\right) b_{M}^{\prime}\left(\phi_{r}(w)\right) \phi_{r}^{\prime}(w) .
\end{aligned}
$$

Evaluating the second condition at $w=\psi_{r}(m)$, multiplying it by $\psi_{r}^{\prime}(m)>0$, and using $\phi_{r}\left(\psi_{r}(m)\right)=m$ and $\phi_{r}^{\prime}\left(\psi_{r}(m)\right) \psi_{r}^{\prime}(m)=1$, we obtain the following system of first-order ODE for $b_{m}$ and $b_{W} \circ \psi_{r}$ :

$$
\begin{aligned}
b_{M}^{\prime}(m) & \left.=\gamma_{M} m \psi_{r}^{\prime}(m)+\delta_{M}^{\prime}\left(\left(b_{W} \circ \psi_{r}\right)(m)\right)\right)\left(b_{W} \circ \psi_{r}\right)^{\prime}(m) \\
\left(b_{W} \circ \psi_{r}\right)^{\prime}(m) & =\gamma_{W} \psi_{r}(m)+\delta_{W}^{\prime}\left(b_{M}(m)\right) b_{M}^{\prime}(m) .
\end{aligned}
$$

In the case $\delta_{M}^{\prime}(0) \delta_{W}^{\prime}(0)<1$, we may rewrite the system (20) equivalently in standard form

$$
\begin{aligned}
b_{M}^{\prime}(m) & =\frac{\gamma_{M} m \psi_{r}^{\prime}(m)+\delta_{M}^{\prime}\left(\left(b_{W} \circ \psi_{r}\right)(m)\right) \gamma_{W} \psi_{r}(m)}{1-\delta_{W}^{\prime}\left(b_{M}(m)\right) \delta_{M}^{\prime}\left(\left(b_{W} \circ \psi_{r}\right)(m)\right)} \\
\left(b_{W} \circ \psi_{r}\right)^{\prime}(m) & =\frac{\gamma_{W} \psi_{r}(m)+\delta_{W}^{\prime}\left(b_{M}(m)\right) \gamma_{M} m \psi_{r}^{\prime}(m)}{1-\delta_{W}^{\prime}\left(b_{M}(m)\right) \delta_{M}^{\prime}\left(\left(b_{W} \circ \psi_{r}\right)(m)\right)} .
\end{aligned}
$$

$\delta_{M}^{\prime}(0) \delta_{W}^{\prime}(0)<1$ and the continuity of $\delta_{M}^{\prime \prime}$ and $\delta_{W}^{\prime \prime}$ imply that the Lipschitz condition guaranteeing a unique pair of continuously differentiable solutions $\left(b_{M}, b_{W} \circ \psi_{r}\right)$ with initial values $b_{M}\left(m_{r}\right)=b_{W}\left(\psi_{r}\left(m_{r}\right)\right)=0$ is satisfied, and clearly both functions are strictly increasing. Sufficiency conditions for optimality follow immediately from supermodularity. The formulas for $u_{M}^{(r)}$ and $u_{W}^{(r)}$ follow from $u_{M}^{(r)}\left(m_{r}\right)=u_{W}^{(r)}(0)=0$ and payoff equivalence. This proves part (i).

For part (ii), note that if $b_{M}$ and $b_{W}$ are strictly increasing functions, they are differentiable almost everywhere. In particular, there is a set of full measure in $\left[m_{r}, \bar{m}\right]$ where both $b_{M}$ and $b_{W} \circ \psi_{r}$ are differentiable. But, if $b_{M}$ and $b_{W}$ are equilibrium strategies, the system (20) must be satisfied for each of these types. However, for $\delta_{M}^{\prime} \equiv 1$ and $\delta_{W}^{\prime} \equiv 1$, (20) is violated for any $m$. This concludes the proof.

In the proofs of Lemma 7 below and Theorems 4 and 5 we use the following well- 
known result about the asymptotic distribution of central order statistics,

Lemma 6. Fix any $m \in(\underline{m}, \bar{m})$ and set $p=F(m)>0$. If $\left(j_{n}\right)_{n \in \mathbb{N}}$ is a sequence that satisfies $\frac{j_{n}}{n}-p=o\left(n^{-1 / 2}\right)$, then

$$
\sqrt{n}\left(M_{j_{n}: n}-m\right) \rightarrow^{d} N\left(0, \frac{p(1-p)}{f(m)^{2}}\right)
$$

where $\rightarrow^{d}$ denotes convergence in distribution, as $n \rightarrow \infty$. In particular, the result applies for $j_{n}=\lceil p n\rceil$, the ceiling of pn (the smallest integer greater than or equal to pn).

Proof of Lemma 6. See Theorem 10.3 in David and Nagaraja (2003).

Lemma 7. i) For all $m \in[0, \bar{m}]$ and $w \in[0, \bar{w}]$ :

$$
\lim _{k \rightarrow \infty, \frac{k}{n_{k}} \rightarrow 1-r} \Psi^{\left(n_{k}, k\right)}(m)=\psi_{r}(m) \text { and } \lim _{k \rightarrow \infty, \frac{k}{n_{k}} \rightarrow 1-r} \Phi^{\left(n_{k}, k\right)}(w)=\phi_{r}(w) .
$$

ii) For all $m \in[0, \bar{m}]$ and $w \in[0, \bar{w}]$ :

$\lim _{k \rightarrow \infty, \frac{k}{n_{k}} \rightarrow 1-r} \int_{0}^{m} \Psi^{\left(n_{k}, k\right)}(s) d s=\int_{0}^{m} \psi_{r}(s) d s$ and $\lim _{k \rightarrow \infty, \frac{k}{n_{k}} \rightarrow 1-r} \int_{0}^{w} \Phi^{\left(n_{k}, k\right)}(s) d s=\int_{0}^{w} \phi_{r}(s) d s$.

Proof of Lemma 7. i) We give the proof for $\lim _{k \rightarrow \infty, \frac{k}{n_{k}} \rightarrow 1} \Psi^{\left(n_{k}, k\right)}(m)=\psi_{0}(m)$, i.e., for the convergence of a given type of man's expected partner when $r=0$. The proof for $r \in(0,1)$ is a bit more cumbersome in terms of notation, but otherwise analogous. The proof for the convergence of $\Phi^{\left(n_{k}, k\right)}$ is analogous as well. Consider any $m \in(0, \bar{m})$ and set $p=F(m)>0$. Fixing an arbitrary $\varepsilon>0$ (with $m-\varepsilon \in(0, \bar{m})$ and $m+\varepsilon \in(0, \bar{m}))$, the convergence in law of $\sqrt{n_{k}}\left(M_{\left\lceil F(m-\varepsilon) n_{k}\right\rceil: n_{k}-1}-(m-\varepsilon)\right.$ ) (by Lemma 6) implies that $F_{\left\lceil F(m-\varepsilon) n_{k}\right\rceil: n_{k}-1}(m)$ converges exponentially fast to 1 (in $k$, or equivalently $n_{k}$ ). Moreover $F_{j: n_{k}-1}(m) \geq F_{\left\lceil F(m-\varepsilon) n_{k}\right\rceil: n_{k}-1}(m)$ for all $j \leq\lceil F(m-\varepsilon) n\rceil$ (by stochastic dominance). Similarly, $F_{\left\lceil F(m+\varepsilon) n_{k}\right\rceil: n_{k}-1}(m)$ converges exponentially fast to 0 , and $F_{j: n_{k}-1}(m) \leq F_{\left\lceil F(m+\varepsilon) n_{k}\right\rceil: n_{k}-1}(m)$ for all $j \geq\left\lceil F(m+\varepsilon) n_{k}\right\rceil$. It follows that

$$
\Psi^{\left(n_{k}, k\right)}(m)= \begin{cases}E\left[W_{1: n_{k}}\right]+\sum_{j=1}^{n_{k}-1} F_{j: n_{k}-1}(m) E\left[W_{j+1: n_{k}}-W_{j: n_{k}}\right] & \text { if } n_{k}=k \\ \sum_{j=0}^{k-1} F_{n_{k}-k+j: n_{k}-1}(m) E\left[W_{j+1: k}-W_{j: k}\right] & \text { if } n_{k}>k\end{cases}
$$

can be written as the sum of a number in $\left[E\left[W_{\left\lceil F(m-\varepsilon) n_{k}\right\rceil+k-n_{k}: k}\right], E\left[W_{\left\lceil F(m+\varepsilon) n_{k}\right\rceil+k-n_{k}: k}\right]\right.$ and an error term that converges exponentially fast to zero. Moreover, as a simple consequence of Lemma 6 (applied to women's order statistics), $\lim _{k \rightarrow \infty, \frac{k}{n_{k}} \rightarrow 1} E\left[W_{\left\lceil F(m-\varepsilon) n_{k}\right\rceil+k-n_{k}: k}\right]=$ 
$G^{-1}(F(m-\varepsilon))$ and $\lim _{k \rightarrow \infty, \frac{k}{n_{k}} \rightarrow 1} E\left[W_{\left\lceil F(m+\varepsilon) n_{k}\right\rceil+k-n_{k}: k}\right]=G^{-1}(F(m+\varepsilon))$. Thus, it follows that

$$
G^{-1}(F(m-\varepsilon)) \leq \liminf _{k \rightarrow \infty, \frac{k}{n_{k}} \rightarrow 1} \Psi^{\left(n_{k}, k\right)}(m) \leq \limsup _{k \rightarrow \infty, \frac{k}{n_{k}} \rightarrow 1} \Psi^{\left(n_{k}, k\right)}(m) \leq G^{-1}(F(m+\varepsilon)) .
$$

Letting $\varepsilon \rightarrow 0$ then yields $\lim _{k \rightarrow \infty, \frac{k}{n_{k}} \rightarrow 1} \Psi^{\left(n_{k}, k\right)}(m)=\psi_{0}(m)$, for all $m \in(0, \bar{m})$. The result for $m=0$ and $m=\bar{m}$ now follows immediately from the monotonicity and boundedness of $\Psi^{\left(n_{k}, k\right)}$.

ii) This follows from (i) and the Dominated Convergence Theorem.

We will use the following two lemmas in the proofs of Theorems 4 and 5. Lemma 8 is due to Varah (1975).

Lemma 8 (Varah, 1975). Let A be a $l \times l$-matrix that is diagonally dominant by rows. That is, for all $i=0, \ldots, l-1,\left|a_{i, i}\right|>\sum_{j \neq i}\left|a_{i, j}\right|$. Set $\alpha:=\min _{i}\left(\left|a_{i, i}\right|-\sum_{j \neq i}\left|a_{i, j}\right|\right)$. Then $\left\|A^{-1}\right\|_{\infty} \leq \frac{1}{\alpha}$.

Lemma 9. $\left\|\Delta_{W}^{a_{W}}\right\|_{\infty}=O\left(\frac{1}{n}\right)$, and if Condition 1 is satisfied also $\left\|\Delta_{M}^{a_{M}}\right\|_{\infty}=O\left(\frac{1}{k}\right)$.

Proof of Lemma 9. Let $c_{f}:=\min _{m \in[\underline{m}, \bar{m}]} f(m)>0$ and $c_{g}:=\min _{w \in[\underline{w}, \bar{w}]} g(w)>0$. We will prove the lemma using the representations of $\Delta_{W}^{a_{W}}$ and $\Delta_{M}^{a_{M}}$ from Lemma 4. Note first that, for all $i \in\{0, \ldots, n-1\}$,

$$
\begin{aligned}
\Delta_{M, i}^{I_{1}} & =E\left[M_{i+1: n}-M_{i: n}\right]=\int_{\underline{m}}^{\bar{m}}\left(F_{i: n}(m)-F_{i+1: n}(m)\right) d m+\mathbb{I}_{\{i=0\}} \underline{m} \\
& =\int_{\underline{m}}^{\bar{m}}\left(\begin{array}{c}
n \\
i
\end{array}\right) F^{i}(m)(1-F(m))^{n-i} d m+\mathbb{I}_{\{i=0\} \underline{m}} \\
& \leq \frac{1}{c_{f}} \int_{\underline{m}}^{\bar{m}}\left(\begin{array}{c}
n \\
i
\end{array}\right) F^{i}(m)(1-F(m))^{n-i} f(m) d m+\mathbb{I}_{\{i=0\} \underline{m}} \\
& =\frac{1}{c_{f}(n+1)}+\mathbb{I}_{\{i=0\} \underline{m}},
\end{aligned}
$$

where $\mathbb{I}_{\{\cdot\}}$ is the usual indicator function. An analogous inequality applies, of course, for $\Delta_{W, i}^{I_{1}}, i \in\{0, \ldots, k-1\}$. Using Lemma 4 and Lemma 2 (ii), we obtain:

$$
\begin{array}{r}
\Delta_{W, i}^{a_{W}}=\sum_{j=1}^{k-1} \theta_{k, i j} E\left[W_{i+j: 2 k-1}\right] \hat{\Delta}_{M, j}^{I_{1}}<\frac{\bar{w}(k-1)}{c_{f}(k+1)(n+1)}=O\left(\frac{1}{n}\right) \\
\Delta_{M, i}^{a_{M}}=\sum_{j=0}^{k-1} \theta_{n, i(n-k+j)} E\left[M_{i+n-k+j: 2 n-1}\right] \Delta_{W, j}^{I_{1}}<\frac{\bar{m}(n-1)}{c_{g}(k+1)(n+1)}=O\left(\frac{1}{k}\right),
\end{array}
$$

where the second inequality uses Condition 1. 
Proof of Theorem 4. Given the results of Lemma 3 and Lemma 7 ii), we still have to show

$$
\lim _{n \rightarrow \infty} E\left[\delta_{M}\left(b_{W}^{(n, n)}\left(W_{1: n}\right)\right)\right]=\lim _{n \rightarrow \infty} E\left[\delta_{W}\left(b_{M}^{(n, n)}\left(M_{1: n}\right)\right)\right]=0 \quad \text { (balanced case) }
$$

and

$$
\lim _{k \rightarrow \infty, k<n_{k}, \frac{k}{n_{k}} \rightarrow 1-r} E\left[\delta_{W}\left(b_{M}^{\left(n_{k}, k\right)}\left(M_{n_{k}-k+1: n_{k}}\right)\right)\right]=0 \quad \text { (unbalanced case). }
$$

As equilibrium strategies for arbitrary benefit functions satisfying $\delta_{M}^{\prime}(0) \delta_{W}^{\prime}(0)<1$ are pointwise dominated by the equilibrium strategies for the case of linear benefit functions with $d_{M}=\delta_{M}^{\prime}(0)$ and $d_{W}=\delta_{W}^{\prime}(0)$ (see the proof of Theorem 6), we need to consider only the latter case. Starting from (11), we obtain:

$$
\begin{aligned}
\left\|\hat{\Delta}_{M}^{b_{M}^{(n, k)}}\right\|_{\infty} & =\left\|\left(I_{k}-d_{M} d_{W} \hat{\Theta}_{n, k} \Theta_{k}\right)^{-1}\left(\gamma_{M} \hat{\Delta}_{M}^{a_{M}^{(n, k)}}+d_{M} \gamma_{W} \hat{\Theta}_{n, k} \Delta_{W}^{a_{W}^{(n, k)}}\right)\right\|_{\infty} \\
& \leq\left\|\left(I_{k}-d_{M} d_{W} \hat{\Theta}_{n, k} \Theta_{k}\right)^{-1}\right\|_{\infty}\left(\gamma_{M}\left\|\hat{\Delta}_{M}^{a_{M}^{(n, k)}}\right\|_{\infty}+d_{M} \gamma_{W}\left\|\hat{\Theta}_{n, k}\right\|_{\infty}\left\|\Delta_{W}^{a_{W}^{(n, k)}}\right\|_{\infty}\right)=O\left(\frac{1}{k}\right)
\end{aligned}
$$

where the last step uses $\left\|\left(I_{k}-d_{M} d_{W} \hat{\Theta}_{n, k} \Theta_{k}\right)^{-1}\right\|_{\infty} \leq \frac{1}{1-d_{M} d_{W}}$ (by Lemma 8 , because $\hat{\Theta}_{n, k} \Theta_{k}$ is sub-stochastic), $\left\|\hat{\Theta}_{n, k}\right\|_{\infty} \leq 1$, as well as $\left\|\hat{\Delta}_{M}^{a_{M}^{(n, k)}}\right\|_{\infty}=O\left(\frac{1}{k}\right)$ and $\left\|\Delta_{W}^{a_{W}^{(n, k)}}\right\|_{\infty}=$ $O\left(\frac{1}{n}\right)$ (by Lemma 9). An analogous argument, starting from (12) shows $\left\|\Delta_{W}^{b_{W}^{(n, k)}}\right\|_{\infty}=$ $O\left(\frac{1}{k}\right)$. As $E\left[b_{W}^{(n, n)}\left(W_{1: n}\right)\right]=\Delta_{W, 0}^{b_{W}^{(n, n)}}$ and $E\left[b_{M}^{(n, n)}\left(M_{1: n}\right)\right]=\Delta_{M, 0}^{b_{M}^{(n, n)}}$, this proves the claim of the Theorem for balanced markets.

It remains to be shown that $\left.\lim _{k \rightarrow \infty, k<n_{k}, \frac{k}{n_{k}} \rightarrow 1-r} E\left[b_{M}^{\left(n_{k}, k\right)}\left(M_{n_{k}-k+1: n_{k}}\right)\right)\right]=0$. Note that

$$
\begin{aligned}
b_{M}^{\left(n_{k}, k\right)}(m) & =\gamma_{M} a_{M}^{\left(n_{k}, k\right)}(m)+d_{M} \hat{\mathscr{F}}(m) \cdot \Delta_{W}^{b_{W}^{\left(n_{k}, k\right)}} \\
& \leq \hat{\mathscr{F}}(m) \cdot\left(\gamma_{M} m \Delta_{W}^{I_{1}}+d_{M} \Delta_{W}^{b_{W}^{\left(n_{k}, k\right)}}\right)=\sum_{j=0}^{k-1} F_{n_{k}-k+j: n_{k}-1}(m)\left(\gamma_{M} m \Delta_{W, j}^{I_{1}}+d_{M} \Delta_{W, j}^{b_{W}^{\left(n_{k}, k\right)}}\right) .
\end{aligned}
$$

As $\left\|\Delta_{W}^{I_{1}}\right\|_{\infty}=O\left(\frac{1}{k}\right)$ (see the proof of Lemma 9) and $\left\|\Delta_{W}^{b_{W}^{(n, k)}}\right\|_{\infty}=O\left(\frac{1}{k}\right)$ (see above), the claim follows immediately from Lemma 6 , which implies that for an arbitrary $\varepsilon>0$ and any sequence $j_{k} \geq \varepsilon k$, the probability that $F_{n_{k}-k+j_{k}: n_{k}-1}\left(M_{n_{k}-k+1: n_{k}}\right)$ is larger than $\varepsilon$ converges exponentially fast to 0 .

The proof of Theorem 5 i) uses the following lemma. 
Lemma 10. i) The diagonal entries of $\Theta_{n}$ satisfy:

$$
\begin{aligned}
\operatorname{argmin}_{i \in\{1, \ldots, n-1\}} \theta_{n, i i} & =\frac{n}{2} \text { if } n \text { is even }, \\
\operatorname{argmin}_{i \in\{1, \ldots, n-1\}} \theta_{n, i i} & =\left\{\frac{n-1}{2}, \frac{n+1}{2}\right\} \text { if } n \text { is odd } .
\end{aligned}
$$

ii) For even $n, \lim _{n \rightarrow \infty} \theta_{n, \frac{n}{2} \frac{n}{2}} \sqrt{\pi n}=1$, and for odd $n, \lim _{n \rightarrow \infty} \theta_{n, \frac{n-1}{2} \frac{n-1}{2}} \sqrt{\pi n}=1$.

Proof of Lemma 10. i) According to Lemma 2 (i), for $i \in\{1, \ldots, n-1\}$ :

$$
\theta_{n, i i}=\frac{n-1}{2 n-1} \frac{\left(\begin{array}{c}
n \\
i
\end{array}\right)\left(\begin{array}{c}
n-2 \\
i-1
\end{array}\right)}{\left(\begin{array}{c}
2 n-2 \\
2 i-1
\end{array}\right)}=\frac{n-1}{2 n-1} \frac{\left(\begin{array}{c}
2 i-1 \\
i
\end{array}\right)\left(\begin{array}{c}
2 n-1-2 i \\
n-i
\end{array}\right)}{\left(\begin{array}{c}
2 n-2 \\
n
\end{array}\right)} .
$$

For $i \in\{1, \ldots, n-2\}$, consider $\ln \theta_{n, i i}-\ln \theta_{n,(i+1)(i+1)}$.

$$
\begin{aligned}
& \ln \theta_{n, i i}-\ln \theta_{n,(i+1)(i+1)}=\left(\sum_{l=i+1}^{2 i-1} \ln l-\sum_{l=1}^{i-1} \ln l+\sum_{l=n+1-i}^{2 n-1-2 i} \ln l-\sum_{l=1}^{n-1-i} \ln l\right) \\
& -\left(\sum_{l=i+2}^{2 i+1} \ln l-\sum_{l=1}^{i} \ln l+\sum_{l=n-i}^{2 n-3-2 i} \ln l-\sum_{l=1}^{n-2-i} \ln l\right)=\ln (i+1)-\ln (2 i) \\
& -\ln (2 i+1)+\ln i-\ln (n-i)+\ln (2 n-2-2 i)+\ln (2 n-1-2 i)-\ln (n-1-i) \\
& =\ln (i+1)-\ln (2 i+1)-\ln (n-i)+\ln (2 n-1-2 i) \\
& =\ln \left(2-\frac{1}{n-i}\right)-\ln \left(2-\frac{1}{i+1}\right) .
\end{aligned}
$$

Thus, if $n$ is even, we have $\theta_{n, i i}>\theta_{n,(i+1)(i+1)}$ if and only if $i \leq \frac{n}{2}-1$ (otherwise the strict reverse inequality holds). The claim for odd $n$ also follows.

ii) This follows from Stirling's approximation, $n !=\sqrt{2 \pi n}\left(\frac{n}{e}\right)^{n}\left(1+O\left(\frac{1}{n}\right)\right)$. We spell out the case of even $n$.

$$
\begin{aligned}
& \sqrt{\pi n} \theta_{n, \frac{n}{2} \frac{n}{2}}=\sqrt{\pi n} \frac{n-1}{2 n-1} \frac{n !(n-2) !(n-1) !^{2}}{\left(\frac{n}{2}\right) !^{2}\left(\frac{n-2}{2}\right) !^{2}(2 n-2) !} \\
& =\sqrt{n} \frac{n-1}{2 n-1} \frac{1}{\sqrt{2}} \frac{\sqrt{n(n-2)}(n-1)}{\frac{n}{2} \frac{n-2}{2} \sqrt{2 n-2}} \frac{n^{n}(n-2)^{n-2}(n-1)^{2 n-2}}{\left(\frac{n}{2}\right)^{n}\left(\frac{n-2}{2}\right)^{n-2}(2 n-2)^{2 n-2}} \frac{\left(1+O\left(\frac{1}{n}\right)\right)}{\left(1+O\left(\frac{1}{n}\right)\right)} \\
& =\sqrt{n} \frac{n-1}{2 n-1} \frac{2 \sqrt{n-1}}{\sqrt{n(n-2)}} \frac{\left(1+O\left(\frac{1}{n}\right)\right)}{\left(1+O\left(\frac{1}{n}\right)\right)} .
\end{aligned}
$$

This ratio converges to 1 as $n \rightarrow \infty$.

Proof of Theorem 5. Proof of part (i) (balanced markets): We show $\lim _{n \rightarrow \infty}\left(E\left[b_{M}^{(n, n)}\left(M_{1: n}\right)\right]-\right.$ $\left.E\left[b_{W}^{(n, n)}\left(W_{1: n}\right)\right]\right)=0$, i.e., $\lim _{n \rightarrow \infty}\left(\Delta_{M, 0}^{b_{M}^{(n, n)}}-\Delta_{W, 0}^{b_{W}^{(n, n)}}\right)=0$. 
Given a vector $v=\left(v_{0}, \ldots, v_{n-1}\right) \in \mathbb{R}^{n}$, we write $v_{-0}$ for the vector $\left(v_{1}, \ldots, v_{n-1}\right)$. We define $V_{n} \in \mathbb{R}^{n}$ via $V_{n, j}:=\theta_{n, 0 j}$ for $j \in\{0, \ldots, n-1\}$, i.e., $V_{n}$ is the first row vector of $\Theta_{n}$. Recall also that $\hat{\Theta}_{n, n-1}$ is the $(n-1) \times(n-1)$ matrix that results from deleting the first column (which has all entries equal to zero) and the first row of $\Theta_{n}$. (9) and (10) imply:

$$
\begin{aligned}
& \Delta_{M, 0}^{b_{M}^{(n, n)}}=\gamma_{M} \Delta_{M, 0}^{a_{M}^{(n, n)}}+V_{n,-0} \cdot \Delta_{W,-0}^{b_{W}^{(n, n)}} \\
& \Delta_{W, 0}^{b_{W}^{(n, n)}}=\gamma_{W} \Delta_{W, 0}^{a_{W}^{(n, n)}}+V_{n,-0} \cdot \Delta_{M,-0}^{b_{M}^{(n, n)}}
\end{aligned}
$$

Thus, $\Delta_{M, 0}^{b_{M}^{(n, n)}}-\Delta_{W, 0}^{b_{W}^{(n, n)}}=\gamma_{M} \Delta_{M, 0}^{a_{M}^{(n, n)}}-\gamma_{W} \Delta_{W, 0}^{a_{W}^{(n, n)}}+V_{n,-0} \cdot\left(\Delta_{W,-0}^{b_{W}^{(n, n)}}-\Delta_{M,-0}^{b_{M}^{(n, n)}}\right)$. As $\left\|\Delta_{M}^{a_{M}^{(n, n)}}\right\|_{\infty}=$ $O\left(\frac{1}{n}\right)$ and $\left\|\Delta_{W}^{a_{W}^{(n, n)}}\right\|_{\infty}=O\left(\frac{1}{n}\right)$ (by Lemma 9), and $\left\|V_{n,-0}\right\|_{1}=\frac{n-1}{n+1}$ (by Lemma 2 ii), $\lim _{n \rightarrow \infty}\left(\Delta_{M, 0}^{b_{M}^{(n, n)}}-\Delta_{W, 0}^{b_{W}^{(n, n)}}\right)=0$ follows from

$$
\lim _{n \rightarrow \infty}\left\|\Delta_{W,-0}^{b_{W}^{(n, n)}}-\Delta_{M,-0}^{b_{M}^{(n, n)}}\right\|_{\infty}=0
$$

which we now show. First, using (again) that the first column of $\Theta_{n}$ is zero, (9) and (10) yield

$$
\begin{aligned}
& \Delta_{M,-0}^{b_{M}^{(n, n)}}=\gamma_{M} \Delta_{M,-0}^{a_{M}^{(n, n)}}+\hat{\Theta}_{n, n-1} \Delta_{W,-0}^{b_{W}^{(n, n)}} \\
& \Delta_{W,-0}^{b_{W}^{(n, n)}}=\gamma_{W} \Delta_{W,-0}^{a_{W}^{(n, n)}}+\hat{\Theta}_{n, n-1} \Delta_{M,-0}^{b_{M}^{(n, n)}}
\end{aligned}
$$

Thus, analogous to (11) and (12), we find the following explicit representations of $\Delta_{M,-0}^{b_{M}^{(n, n)}}$ and $\Delta_{W,-0}^{b_{W}^{(n, n)}}$ (using only the entries of $\left.\hat{\Theta}_{n, n-1}\right)$ :

$$
\begin{aligned}
\Delta_{M,-0}^{b_{M}^{(n, n)}} & =\left(I_{n-1}-\hat{\Theta}_{n, n-1}^{2}\right)^{-1}\left(\gamma_{M} \Delta_{M,-0}^{a_{M}^{(n, n)}}+\gamma_{W} \hat{\Theta}_{n, n-1} \Delta_{W,-0}^{a_{W}^{(n, n)}}\right) \\
\Delta_{W,-0}^{b_{W}^{(n, n)}} & =\left(I_{n-1}-\hat{\Theta}_{n, n-1}^{2}\right)^{-1}\left(\gamma_{W} \Delta_{W,-0}^{a_{W}^{(n, n)}}+\gamma_{M} \hat{\Theta}_{n, n-1} \Delta_{M,-0}^{a_{M}^{(n, n)}}\right) .
\end{aligned}
$$

It follows that

$$
\begin{aligned}
\left\|\Delta_{W,-0}^{b_{W}^{(n, n)}}-\Delta_{M,-0}^{b_{M}^{(n, n)}}\right\|_{\infty} & =\left\|\left(I_{n-1}-\hat{\Theta}_{n, n-1}^{2}\right)^{-1}\left(I_{n-1}-\hat{\Theta}_{n, n-1}\right)\left(\gamma_{W} \Delta_{W,-0}^{a_{W}^{(n, n)}}-\gamma_{M} \Delta_{M,-0}^{a_{M}^{(n, n)}}\right)\right\|_{\infty} \\
& =\left\|\left(I_{n-1}+\hat{\Theta}_{n, n-1}\right)^{-1}\left(\gamma_{W} \Delta_{W,-0}^{a_{W, n}^{(n, n)}}-\gamma_{M} \Delta_{M,-0}^{a_{M}^{(n, n)}}\right)\right\|_{\infty} \\
& \leq\left\|\left(I_{n-1}+\hat{\Theta}_{n, n-1}\right)^{-1}\right\|_{\infty}\left(\gamma_{W}\left\|\Delta_{W,-0}^{a_{W}^{(n, n)}}\right\|_{\infty}+\gamma_{M}\left\|\Delta_{M,-0}^{a_{M}^{(n, n)}}\right\|_{\infty}\right) .
\end{aligned}
$$


But, $\left\|\left(I_{n-1}+\hat{\Theta}_{n, n-1}\right)^{-1}\right\|_{\infty}=O(\sqrt{n})$. Indeed, from Lemma 2 (ii), we know that $\min _{i \in\{1, \ldots, n-1\}}(1+$ $\left.\theta_{n, i i}-\sum_{j \neq i} \theta_{n, i j}\right)=\min _{i \in\{1, \ldots, n-1\}}\left(2 \theta_{n, i i}+\frac{2}{n+1}\right)$. Hence, Lemma 10 implies

$$
\lim _{n \rightarrow \infty}\left(\frac{\sqrt{\pi n}}{2} \min _{i \in\{1, \ldots, n-1\}}\left(1+\theta_{n, i i}-\sum_{j \neq i} \theta_{n, i j}\right)\right)=1 .
$$

Thus, by Lemma $8, \limsup _{n \rightarrow \infty} \frac{2\left\|\left(I_{n-1}+\hat{\Theta}_{n, n-1}\right)^{-1}\right\|_{\infty}}{\sqrt{\pi n}} \leq 1$, so that $\left\|\left(I_{n-1}+\hat{\Theta}_{n, n-1}\right)^{-1}\right\|_{\infty}=$ $O(\sqrt{n})$. Using $\left\|\Delta_{M}^{a_{M}^{(n, n)}}\right\|_{\infty}=O\left(\frac{1}{n}\right)$ and $\left\|\Delta_{W}^{a_{W}^{(n, n)}}\right\|_{\infty}=O\left(\frac{1}{n}\right)$, it follows that $\| \Delta_{W,-0}^{b_{W}^{(n, n)}}-$ $\Delta_{M,-0}^{b_{M}^{(n, n)}} \|_{\infty}=O\left(\frac{1}{\sqrt{n}}\right)$.

Proof of part (ii) (unbalanced markets): Analogous to the argument in the main text, the sum of all agents' ex-ante expected utilities is bounded by ex-ante expected aggregate match surplus:

$$
\begin{aligned}
& E\left[\frac{1}{k} \sum_{i=1}^{k} M_{n_{k}-k+i: n_{k}} W_{i: k}\right]>\frac{n_{k}}{k} \int_{0}^{\bar{m}} U_{M}^{\left(n_{k}, k\right)}(m) f(m) d m+\int_{0}^{\bar{w}} U_{W}^{\left(n_{k}, k\right)}(w) g(w) d w \\
& =\gamma_{M} \int_{0}^{\bar{m}} \int_{0}^{m} \Psi^{\left(n_{k}, k\right)}(s) d s f(m) \frac{n_{k}}{k} d m+E\left[b_{M}\left(M_{n_{k}-k+1: n_{k}}\right)\right]+\gamma_{W} \int_{0}^{\bar{w}} \int_{0}^{w} \Phi^{\left(n_{k}, k\right)}(s) d s g(w) d w .
\end{aligned}
$$

The strict inequality in the bound above is due to the fact that the investments of the men who fail to match are lost. Note that $\lim _{k \rightarrow \infty, k<n_{k}, \frac{k}{n_{k}} \rightarrow 1-r} E\left[\frac{1}{k} \sum_{i=1}^{k} M_{n_{k}-k+i: n_{k}} W_{i: k}\right]=$ $S^{(r)}$ (by the LLN for empirical distributions), $\lim _{k \rightarrow \infty, k<n_{k}, \frac{k}{n_{k}} \rightarrow 1-r} \int_{0}^{\bar{m}} \int_{0}^{m} \Psi^{\left(n_{k}, k\right)}(s) d s f(m) \frac{n_{k}}{k} d m=$ $R_{M}^{(r)}$ and $\lim _{k \rightarrow \infty, k<n_{k}, \frac{k}{n_{k}} \rightarrow 1-r} \int_{0}^{\bar{w}} \int_{0}^{w} \Phi^{\left(n_{k}, k\right)}(s) d s g(w) d w=R_{W}^{(r)}$ (by Lemma 7 and the Dominated Convergence Theorem), and $S^{(r)}=R_{M}^{(r)}+R_{W}^{(r)}$ (as $R_{M}^{(r)}$ and $R_{W}^{(r)}$ are the aggregate core utilities in the continuum model). Thus, $\limsup _{k \rightarrow \infty, k<n_{k}, \frac{k}{n_{k}} \rightarrow 1-r} E\left[b_{M}\left(M_{n_{k}-k+1: n_{k}}\right)\right] \leq$ $\gamma_{W} R_{M}^{(r)}+\gamma_{M} R_{W}^{(r)}$. We show now that $\lim _{k \rightarrow \infty, k<n_{k}, \frac{k}{n_{k}} \rightarrow 1-r} E\left[b_{M}\left(M_{n_{k}-k+1: n_{k}}\right)\right]=\gamma_{W} R_{M}^{(r)}+$ $\gamma_{M} R_{W}^{(r)}$, i.e., the fraction of the difference between aggregate surplus and aggregate information rents that is dissipated converges to 0 . Indeed, by the above observations, $E\left[b_{M}\left(M_{n_{k}-k+1: n_{k}}\right)\right]=O(1)$ (in the considered limit). Moreover, for any $\varepsilon>0$ and any sequence $j_{k} \geq \varepsilon k, E\left[b_{M}\left(M_{n_{k}-k-j_{k}: n_{k}}\right)\right]$ converges to zero exponentially fast (e.g., because, by Lemma 6 , the probability that $F_{n_{k}-k: n_{k}-1}\left(M_{n_{k}-k-j_{k}: n_{k}}\right)$ is greater than $\varepsilon$ declines exponentially). Thus, all investments that are wasted in expectation, $E\left[b_{M}\left(M_{1: n_{k}}\right)\right], \ldots, E\left[b_{M}\left(M_{n_{k}-k: n_{k}}\right)\right.$ are at most of order 1 , and, for any $\varepsilon>0$, at most $\varepsilon k$ of these are not exponentially small. Consequently, the per-capita expected utility that is lost converges to zero.

Proof of Theorem 6. Consider the equilibrium strategies $\widetilde{b}_{M}$ and $\widetilde{b}_{W}$ for the case of linear benefit functions $\widetilde{\delta}_{M}\left(\beta_{W}\right):=\delta_{M}^{\prime}(0) \beta_{W}$ and $\widetilde{\delta}_{W}\left(\beta_{M}\right):=\delta_{W}^{\prime}(0) \beta_{M}$ (the equilib- 
rium exists, by Theorem 1 , because $\left.r(n, k) \delta_{M}^{\prime}(0) \delta_{W}^{\prime}(0)<1\right)$. The limit conditions on type supports imply $\widetilde{b}_{M}(\bar{m}) \rightarrow 0$ and $\widetilde{b}_{W}(\bar{w}) \rightarrow 0$. Indeed, if $n=k$, then $a_{M}(\bar{m}) \leq$ $\bar{m}(\bar{w}-\underline{w})$ and $a_{W}(\bar{w}) \leq(\bar{m}-\underline{m}) \bar{w}$, and if $n>k$, then $a_{M}(\bar{m}), a_{W}(\bar{w}) \leq \overline{m w}$. Thus, $a_{M}(\bar{m}) \rightarrow 0$ and $a_{W}(\bar{w}) \rightarrow 0$, so that $\Delta_{M}^{a_{M}}, \Delta_{W}^{a_{W}} \rightarrow 0$. Thus, using (12), it follows that $\Delta_{W}^{\widetilde{b}_{W}}=\left(I_{k}-\delta_{M}^{\prime}(0) \delta_{W}^{\prime}(0) \Theta_{k} \hat{\Theta}_{n, k}\right)^{-1}\left(\gamma_{W} \Delta_{W}^{a_{W}}+d_{W} \gamma_{M} \Theta_{k} \hat{\Delta}_{M}^{a_{M}}\right) \rightarrow 0\left(\Theta_{k}\right.$ and $\hat{\Theta}_{n, k}$ are fix $)$. Similarly, $\Delta_{M}^{\widetilde{b}_{M}} \rightarrow 0$ (using (11)). Hence, $\widetilde{b}_{M}(\bar{m})=\gamma_{M} a_{M}(\bar{m})+\delta_{M}^{\prime}(0) \hat{\mathscr{F}}(\bar{m}) \cdot \Delta_{W}^{\widetilde{b}_{W}} \rightarrow 0$, and $\widetilde{b}_{W}(\bar{w})=\gamma_{W} a_{W}(\bar{w})+\delta_{W}^{\prime}(0) \mathscr{G}(\bar{w}) \cdot \hat{\Delta}_{M}^{\widetilde{b}_{M}} \rightarrow 0$.

To complete the proof of the Theorem, we show that, for any equilibrium of the matching contest with benefit functions $\delta_{M}$ and $\delta_{W}$, it holds that $b_{M}(m) \leq \widetilde{b}_{M}(m)$ for all $m \in[\underline{m}, \bar{m}]$ and $b_{W}(w) \leq \widetilde{b}_{W}(w)$ for all $w \in[\underline{w}, \bar{w}]$. First, the concavity of $\delta_{W}$ and $\delta_{M}$, the mean value theorem, and the identities (6) and (7) imply the following bounds for $\hat{\Delta}_{M}^{\delta_{W} \circ b_{M}}$ and $\Delta_{W}^{\delta_{M} \circ b_{W}}$ :

$$
\begin{aligned}
& \hat{\Delta}_{M}^{\delta_{W} \circ b_{M}} \leq \delta_{W}^{\prime}(0)\left(\gamma_{M} \hat{\Delta}_{M}^{a_{M}}+\hat{\Theta}_{n, k} \Delta_{W}^{\delta_{M} \circ b_{W}}\right) \\
& \Delta_{W}^{\delta_{M} \circ b_{W}} \leq \delta_{M}^{\prime}(0)\left(\gamma_{W} \Delta_{W}^{a_{W}}+\Theta_{k} \hat{\Delta}_{M}^{\delta_{W}^{\circ} b_{M}}\right) .
\end{aligned}
$$

Thus (as all entries of $\Theta_{k}$ are non-negative),

$$
\left(I_{k}-\delta_{M}^{\prime}(0) \delta_{W}^{\prime}(0) \Theta_{k} \hat{\Theta}_{n, k}\right) \Delta_{W}^{\delta_{M} \circ b_{W}} \leq \delta_{M}^{\prime}(0)\left(\gamma_{W} \Delta_{W}^{a_{W}}+\delta_{W}^{\prime}(0) \gamma_{M} \Theta_{k} \hat{\Delta}_{M}^{a_{M}}\right)
$$

Applying $\left(I_{k}-\delta_{M}^{\prime}(0) \delta_{W}^{\prime}(0) \Theta_{k} \hat{\Theta}_{n, k}\right)^{-1}=\sum_{l=0}^{\infty}\left(\delta_{M}^{\prime}(0) \delta_{W}^{\prime}(0) \Theta_{k} \hat{\Theta}_{n, k}\right)^{l} \geq 0$ to both sides of the vector inequality (21) yields

$$
\Delta_{W}^{\delta_{M} \circ b_{W}} \leq \delta_{M}^{\prime}(0) \Delta_{W}^{\widetilde{b}_{W}}=\Delta_{W}^{\widetilde{\delta}_{M} \circ \widetilde{b}_{W}} .
$$

$\hat{\Delta}_{M}^{\delta_{W} \circ b_{M}} \leq \hat{\Delta}_{M}^{\widetilde{\delta}_{W} \circ \widetilde{b}_{M}}$ follows from an entirely analogous argument. $b_{M}(m) \leq \widetilde{b}_{M}(m)$ now follows from (4), and $b_{W}(w) \leq \widetilde{b}_{W}(w)$ follows from (5).

Proof of Theorem 7. Consider the expression (6) for $\Delta_{M, i}^{\delta_{W} \circ b_{M}}(i \in\{0, \ldots, n-1\})$. Invoking the concavity of $\delta_{W}$ and the mean value theorem, we obtain the following lower bound:

$$
\Delta_{M, i}^{\delta_{W} \circ b_{M}} \geq \delta_{W}^{\prime}\left(b_{M}(\bar{m})\right)\left(\gamma_{M} \Delta_{M, i}^{a_{M}}+\left(\Theta_{n, k} \Delta_{W}^{\delta_{M} \circ b_{W}}\right)_{i}\right) .
$$

Analogously, (7), the concavity of $\delta_{M}$, and the mean value theorem imply

$$
\Delta_{W, i}^{\delta_{M} \circ b_{W}} \geq \delta_{M}^{\prime}\left(b_{W}(\bar{w})\right)\left(\gamma_{W} \Delta_{W, i}^{a_{W}}+\left(\Theta_{k} \hat{\Delta}_{M}^{\delta_{W} \circ b_{M}}\right)_{i}\right)
$$


for all $i \in\{0, \ldots, k-1\}$. Thus,

$$
\begin{aligned}
& \hat{\Delta}_{M}^{\delta_{W} \circ b_{M}} \geq \delta_{W}^{\prime}\left(b_{M}(\bar{m})\right)\left(\gamma_{M} \hat{\Delta}_{M}^{a_{M}}+\hat{\Theta}_{n, k} \Delta_{W}^{\delta_{M} \circ b_{W}}\right), \\
& \Delta_{W}^{\delta_{M} \circ b_{W}} \geq \delta_{M}^{\prime}\left(b_{W}(\bar{w})\right)\left(\gamma_{W} \Delta_{W}^{a_{W}}+\Theta_{k} \hat{\Delta}_{M}^{\delta_{W} \circ b_{M}}\right) .
\end{aligned}
$$

As all entries of $\Theta_{k}$ are non-negative, we get:

$$
\begin{aligned}
& \left(I_{k}-\delta_{M}^{\prime}\left(b_{W}(\bar{w})\right) \delta_{W}^{\prime}\left(b_{M}(\bar{m})\right) \Theta_{k} \hat{\Theta}_{n, k}\right) \Delta_{W}^{\delta_{M} \circ b_{W}} \\
& \quad \geq \delta_{M}^{\prime}\left(b_{W}(\bar{w})\right)\left(\gamma_{W} \Delta_{W}^{a_{W}}+\delta_{W}^{\prime}\left(b_{M}(\bar{m})\right) \gamma_{M} \Theta_{k} \hat{\Delta}_{M}^{a_{M}}\right) .
\end{aligned}
$$

If $\delta_{M}^{\prime}\left(b_{W}(\bar{w})\right)=0$, then the claim of the theorem holds trivially. Otherwise, the right hand side of (22) is a positive vector. Thus, $\Delta_{W}^{\delta_{M} \circ b_{W}}$ solves

$$
\left(I_{k}-\delta_{M}^{\prime}\left(b_{W}(\bar{w})\right) \delta_{W}^{\prime}\left(b_{M}(\bar{m})\right) \Theta_{k} \hat{\Theta}_{n, k}\right) \Delta_{W}^{\delta_{M} \circ b_{W}}=z
$$

for some $z>0$. In the proof of Theorem 1, we have shown that this is possible if and only if $r(n, k) \delta_{M}^{\prime}\left(b_{W}(\bar{w})\right) \delta_{W}^{\prime}\left(b_{M}(\bar{m})\right)<1$.

\section{References}

[1] Bhaskar, V., and E. Hopkins (2016): "Marriage as a Rat Race: Noisy Pre-Marital Investments with Assortative Matching," Journal of Political Economy 124, 9921045 .

[2] Boas, F. (1897) Kwakiutl Ethnography (ed) H. Codere. Chicago: University Press (1966).

[3] Che, Y.-K., and D. B. Hausch (1999) "Cooperative Investments and the Value of Contracting," The American Economic Review 89(1), 125-147.

[4] Cole, H. L., G. J. Mailath, and A. Postlewaite (2001a): "Efficient NonContractible Investments in Large Economies," Journal of Economic Theory 101, 333-373.

[5] Cole, H. L., G. J. Mailath, and A. Postlewaite (2001b): "Efficient NonContractible Investments in Finite Economies," Advances in Theoretical Economics 1, Iss. 1, Article 2.

[6] David, H. A., and H. N. Nagaraja: Order Statistics, Wiley-Interscience, 2003. 
[7] Dizdar, D. (2017): “Two-sided Investment and Matching with Multidimensional Cost Types and Attributes," forthcoming American Economic Journal: Microeconomics.

[8] Ewerhart, C. (2013): "Regular type distributions in mechanism design and $\rho$ concavity, " Economic Theory 53(3), 591-603.

[9] Felli, L., and K. Roberts (2016): "Does Competition Solve the Hold-Up Problem?” Economica 83, 172-200.

[10] Gregory, C.A. (1980): "Gifts to Men and Gifts to God: Exchange and Capital Accumulation in Contemporary Papua" Man (New Series) 15(4), 626-652.

[11] Hopkins, E. (2012): “Job Market Signaling Of Relative Position, Or Becker Married To Spence," Journal of the European Economic Association 10, 290-322.

[12] Hoppe, H. C., B. Moldovanu, and A. Sela (2009): “The Theory of Assortative Matching Based on Costly Signals," Review of Economic Studies 76, 253-281.

[13] Horn, R. A., and C. R. Johnson: Matrix Analysis, Cambridge University Press, 2013.

[14] Konrad, K. A. (2007): “Strategy in Contests - An Introduction,”Discussion Paper SP II 2007-01, Berlin Wissenschaftszentrum.

[15] Lazear, E. P., and S. Rosen (1981): "Rank-Order Tournaments as Optimum Labor Contracts," Journal of Political Economy 89, 841-864.

[16] Mauss, M. (1935): The Gift. London: Routledge \& Kegan Paul (1974).

[17] Nöldeke, G., and L. Samuelson (2015): "Investment and Competitive Matching," Econometrica 83, 835-896.

[18] Peters, M. (2007): “The Pre-marital investment game," Journal of Economic Theory $137,186-213$.

[19] Peters, M. (2011): “A Non-cooperative approach to Hedonic Equilibrium ” Report, University of British Columbia.

[20] Olszewski, W. and R. Siegel (2016): "Large Contests," Econometrica 84(2), 835854.

[21] Reiss, R.-D.: Approximate Distributions of Order Statistics, Springer, New York, 1989. 
[22] Spence, M. (1973): “Job Market Signaling," Quarterly Journal of Economics 87, 296-332.

[23] Szech, N. (2011): “Optimal Advertising of Auctions, "Journal of Economic Theory $\mathbf{1 4 6}(6), 2596-2607$.

[24] Varah, J. M. (1975): "A Lower Bound for the Smallest Singular Value of a Matrix," Linear Algebra and its Applications 11, 3-5. 


\section{Working Paper Series in Economics}

recent issues

No. 109 Deniz Dizdar, Benny Moldovanu and Nora Szech: The multiplier effect in two-sided markets with bilateral investments, November 2017

No. 108 Andranik S. Tangian: Policy representation by the 2017 Bundestag, September 2017

No. 107 Andranik S. Tangian: Policy representation by German parties at the 2017 federal election, September 2017

No. 106 Andranik S. Tangian: Design and results of the third vote experiment during the 2017 election of the Karlsruhe Institute of Technology student parliament, September 2017

No. 105 Markus Fels: Incentivizing efficient utilization without reducing access: The case against cost-sharing in insurance, July 2017

No. 104 Andranik S. Tangian: Declining labor-labor exchange rates as a cause of inequality growth, July 2017

No. 103 Konstanze Albrecht, Florentin Krämer and Nora Szech: Animal welfare and human ethics: A personality study, June 2017

No. 102 Jannis Engel and Nora Szech: A little good is good enough: Ethical consumption, cheap excuses, and moral self-licensing, March 2017

No. 101 David Freeman, Erik O. Kimbrough, J. Philipp Reiss: Opportunity cost, inattention and the bidder's curse, February 2017

No. 100 Andranik S. Tangian: Selection of questions for VAAs and the VAA-based elections, January 2017

No. 99 Dominik Rothenhäusler, Nikolaus Schweizer and Nora Szech: Guilt in Voting and Public Good Games, November 2016 